\title{
Non-Gaussianity of entanglement entropy and correlations of composite operators
}

\author{
Satoshi Iso, ${ }^{1,2, *}$ Takato Mori, ${ }^{1,2, \dagger}$ and Katsuta Sakai ${ }^{1, \%}$ \\ ${ }^{1}$ KEK Theory Center, High Energy Accelerator Research Organization (KEK), \\ Oho 1-1, Tsukuba, Ibaraki 305-0801, Japan \\ ${ }^{2}$ The Graduate University for Advanced Studies (SOKENDAI), \\ Oho 1-1, Tsukuba, Ibaraki 305-0801, Japan
}

(Received 8 May 2021; accepted 26 May 2021; published 22 June 2021)

\begin{abstract}
This is an extended version of the previous paper [S. Iso et al., Phys. Rev. D 103, 105010 (2021)] to study entanglement entropy (EE) of a half space in interacting field theories. In the previous paper, we have proposed a novel method to calculate EE based on the notion of $\mathbb{Z}_{M}$ gauge theory on Feynman diagrams, and shown that EE consists of two particular contributions, one from a renormalized two-point correlation function in the two-particle irreducible (2PI) formalism and another from interaction vertices. In this paper, we further investigate them in more general field theories and show that the non-Gaussian contributions from vertices can be interpreted as renormalized correlation functions of composite operators.
\end{abstract}

DOI: 10.1103/PhysRevD.103.125019

\section{INTRODUCTION}

Over the past few decades, entanglement entropy (EE) has attracted much interest in various fields. Originally it is proposed as a nice measure for bipartite entanglement within a pure state and has been widely discussed in the context of quantum information. However, its usefulness is not limited to quantum information and its usage in physics is very rich and nontrivial. In condensed matter physics and lattice quantum field theories, EE is a candidate of order parameters to describe quantum phase transition and topological orders for its nonlocality [1-9]. In the context of quantum gravity, the Ryu-Takayanagi formula [10-12], one particular realization of the holography, relates EE of a boundary theory to the extremal surface in the bulk. Furthermore, the information paradox of black holes has been studied in terms of the fine-grained entropy or EE [13-16]. As EE captures quantum correlation, it is also used to investigate decoherence in cosmology [17].

Particularly, EE is a useful quantitative measure for correlations between two spatially separated regions in a ground state. Despite its significance, practical computations of EE in field theories are not an easy task. If we can

\footnotetext{
*satoshi.iso@kek.jp

†moritaka@post.kek.jp

"sakaika@ post.kek.jp
}

Published by the American Physical Society under the terms of the Creative Commons Attribution 4.0 International license. Further distribution of this work must maintain attribution to the author(s) and the published article's title, journal citation, and DOI. Funded by SCOAP . exactly diagonalize the reduced density matrix, the EE can be obtained in a straightforward manner, but such a diagonalization is not generically possible in quantum field theories (QFTs). Thus, EE has been intensively discussed in two particular classes of theories, conformal field theories (CFTs) and Gaussian (i.e., free) theories.

For the former class of theories, we can make the most of the conformal symmetry and many quantities of interest can be determined by their responses to the conformal transformations [6,18-20]. In QFTs which can be treated as perturbations from CFTs, these tools are still available [21-23]. Alternatively, EE of CFTs with gravity duals is calculated using holography, leading to its geometrical interpretation $[10,11]$. (see $[24,25]$ for a review).

On the other hand, for the latter class of theories, the density matrix of the vacuum is Gaussian and in principle, we can perform an explicit calculation [26-29]. There are several ways to deal with it, as summarized for example in $[25,30]$. In the Gaussian case, we can also evaluate EE for a curved boundary or in a curved space by the heat kernel method [13,29,31]. Fermionic extensions are also studied [32].

In comparison to the above two particular classes of theories, we have little understanding of EE for general interacting QFTs, apart from exactly solvable cases [33]. In some supersymmetric theories, the localization method enables an exact calculation of the free energy and EE, [24,25,34-36]. EE in interacting theories also discussed in perturbative [37,38], nonperturbative [39-44] or lattice [45-49] approaches. Nonperturbative studies have taken advantage of the large- $N$ analysis and the renormalization group (RG) flow in the $O(N)$ vector model [39,40,50,51] or 
variational trial wave functions [41-43] or instanton formalism [44]. These works have partly grasped the behavior of EE relevant to renormalization and beyond free theories. Despite these studies, there are many issues yet to be understood.

Interactions generally bring about two important consequences in QFTs: renormalization of IR quantities and non-Gaussianity of the vacuum wave function. Therefore, it is important in the studies of $\mathrm{EE}$ to disentangle these two essentially different effects of interactions. Renormalization is, of course, related to the UV divergences of field theories, but note that UV divergences of EE are already present in free theories. It is simply because there are infinitely many microscopic degrees of freedom that contribute to EE. This type of UV divergences should be regularized by suitably renormalizing parameters in the background gravity [52-57]. In addition, further renormalizations are necessary for interacting theories. In our previous paper [58] we showed that the Gaussian part of EE is given by a renormalized two-point correlation function in the two-particle irreducible (2PI) formalism [59,60] and thus UV divergences specific to interactions are appropriately taken into account. But it is not the end of the story of $\mathrm{EE}$ in interacting theories. Besides renormalization of IR quantities, interactions induce non-Gaussianity of the vacuum wave function and we wonder how much $\mathrm{EE}$ is affected by such non-Gaussianity. Non-Gaussiainity of the vacuum can be expressed in terms of the connected multipoint correlation functions (due to the Wick theorem), and thus the non-Gaussian contributions to EE must be written in terms of higher point Green functions. In the analysis of the $\phi^{4}$ scalar theory in [58], by extracting the Gaussian contributions to EE in 2PI formalism, we have uncovered purely non-Gaussian contributions from fourpoint vertex functions.

In this paper, we continue the investigations of $\mathrm{EE}$ proposed in the previous paper [58] and study further issues of renormalization and non-Gaussianity in a general field-theoretical approach. Especially we will show that the purely non-Gaussian contributions associated with the four-point vertex functions can be interpreted as contributions from renormalized two-point correlation functions of composite operators. The result indicates that all the nonGaussian contributions to EE can be interpreted as a sum of contributions from renormalized two-point correlation functions of various composite operators. We also show that the analysis is not restricted to scalar field theories but applicable to general QFTs with nonzero spins.

The paper is organized as follows. In Sec. II, we give a brief review of the orbifold method. The calculation of EE in a free field theory is demonstrated and a generalization to higher spin fields is commented. In Sec. III, we lift the method to interacting cases and provide a general methodology, $\mathbb{Z}_{M}$ gauge theory on Feynman diagrams, to compute the free energy and EE. We then prove the area law of $\mathrm{EE}$ for interacting cases in the framework. Section IV is devoted to the analysis of contributions to EE from propagators with more detailed explanations and discussions on the calculations in [58]. We introduce the two-particle irreducible (2PI) formalism and express the Gaussian part of EE in terms of the renormalized propagator. Next, we move to the investigation of the nonGaussian contributions in Sec. V. We see that interaction vertices have contributions as well as the Gaussian contributions. We show that these non-Gaussian contributions can be understood as renormalized two-point correlations in terms of the corresponding composite operators. We also discuss an extension to a general theory with spins in Sec. VI. Finally, we make a summary and discussion toward future studies in Sec. VII.

\section{REPLICA TRICK AND ORBIFOLD METHOD}

First, we review the replica trick and the orbifold method to calculate EE. Consider a Hilbert space which consists of two subspaces corresponding to the physical subsystems of interest $A$ and $\bar{A}: \mathcal{H}_{\text {tot }}=\mathcal{H}_{A} \otimes \mathcal{H}_{\bar{A}}$. The EE of $A$ is defined as

$$
S_{A}=-\operatorname{Tr}_{A} \rho_{A} \log \rho_{A},
$$

where $\rho_{A}=\operatorname{Tr}_{\bar{A}} \rho_{\text {tot }}$ is a reduced density matrix of the total one, $\rho_{\text {tot }}$. One of the standard methods to calculate EE in QFTs is known as the replica method [5,6]:

$$
S_{A}:=\lim _{n \rightarrow 1}\left[\frac{1}{1-n} \log \operatorname{Tr} \rho_{A}^{n}\right]=-\lim _{n \rightarrow 1} \frac{\partial}{\partial n}\left[\operatorname{Tr}_{A} \rho_{A}^{n}\right] .
$$

Note that for EE to be uniquely determined by the replica trick, we assume the analytical continuation of $n \in \mathbb{Z}_{>1}$ to $\mathbb{R}$. This formula holds for general $A$ and $\rho$ as long as such an analytical continuation exists. In this paper, we take $A$ as a half space on a time slice in a $(d+1)$ dimensional spacetime, $A=\left\{x^{0}=0, x_{\perp} \geq 0, \forall x_{\|}\right\}$, where $x_{0}$ is a Lorentzian temporal coordinate while $x_{\perp}$ is a one-dimensional normal direction and $x_{\|}$are the rest $(d-1)$-dimensional parallel directions to $\partial A$ (Fig. 1).

Let us define an unnormalized density matrix $\tilde{\rho}_{\text {tot }}$ such that $\rho_{\text {tot }}:=\tilde{\rho}_{\text {tot }} / Z_{1}$, where $Z_{1}$ is a partition function of the

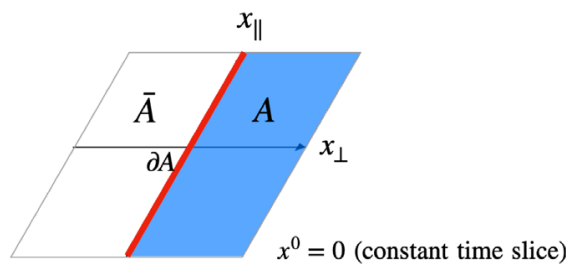

FIG. 1. Our choice of the subregion $A$ and its complement $\bar{A}$. It is a half space given by $A=\left\{x^{0}=0, x_{\perp} \geq 0, \forall x_{\|}\right\}$. The boundary of the subregion is parametrized as $\partial A=\left\{x^{0}=0, x_{\perp}=0, \forall x_{\|}\right\}$. 

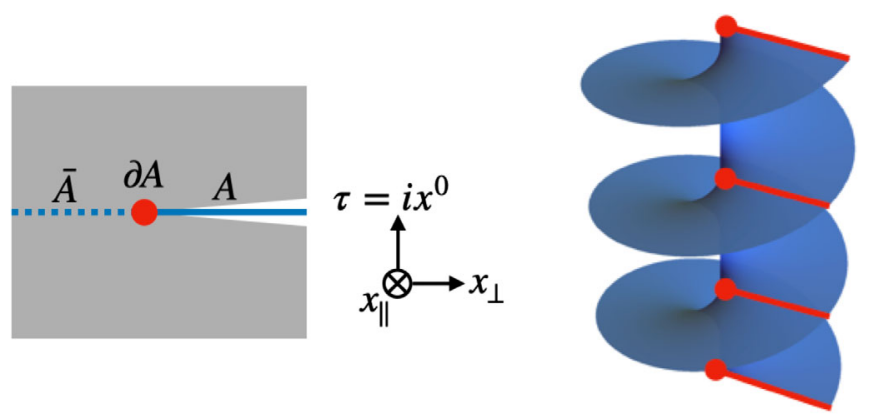

FIG. 2. The Euclidean path integral representation of our reduced density matrix $\tilde{\rho}_{A}$ (left) and its $n$-fold cover $\tilde{\rho}_{A}^{n}(n=3)$ (right).

total system on $\mathbb{R}^{2} \times \mathbb{R}^{d-1}$, where $\mathbb{R}^{2}$ is spanned by two normal coordinates, the Euclidean time $\tau=i x^{0}$ and $x_{\perp}$, with respect to $\partial A$ and the rest $\mathbb{R}^{d-1}$ is spanned by $x_{\|}$. Then, with an unnormalized reduced density matrix defined by $\tilde{\rho}_{A}:=\operatorname{Tr}_{A} \tilde{\rho}_{\text {tot }}, \operatorname{Tr} \tilde{\rho}_{A}^{n}$ is regarded as a partition function of the theory on $\Sigma_{n} \times \mathbb{R}^{d-1}$, where $\Sigma_{n}$ is an $n$-folded cover of a two-dimensional plane spanned by $\tau$ and $x_{\perp}$ or equivalently a two-dimensional cone with a deficit angle $2 \pi(1-n)$. The Euclidean path integral representation of the reduced density matrix and the replicated one is shown in Fig. 2. The EE can be rewritten as

$$
S_{A}=\left.\frac{\partial F_{n}}{\partial n}\right|_{n \rightarrow 1}-F_{1}
$$

in terms of the free energy on $\Sigma_{n} \times \mathbb{R}^{d-1}$

$$
F_{n}:=-\log \operatorname{Tr}_{A} \tilde{\rho}_{A}^{n}
$$

We can further proceed to reduce the calculation employing the orbifold method [61,62]. In this method, we analytically continue $n$ to $1 / M$ with an integer $M$ to obtain the theory on the orbifold $\mathbb{R}^{2} / \mathbb{Z}_{M} \times \mathbb{R}^{d-1}$ instead of a cone. Equation (2) is then rewritten in terms of the free energy $F^{(M)}=F_{1 / n}$ as

$$
S_{A}=-\left.\frac{\partial\left(M F^{(M)}\right)}{\partial M}\right|_{M \rightarrow 1},
$$

provided that $M \in \mathbb{Z}_{>1}$ can be analytically continued to 1 . A state on the orbifold can be obtained with the $\mathbb{Z}_{M}$ projection operator [63],

$$
\hat{P}=\sum_{m=0}^{M-1} \frac{\hat{g}^{m}}{M},
$$

acting on a state in an ordinary flat plane, where $\hat{g}$ is a $2 \pi / M$ rotation operator around the origin,

$$
\hat{g}\left|x, \bar{x}, x_{\|}\right\rangle=\left|e^{2 \pi i / M} x, e^{-2 \pi i / M} \bar{x}, x_{\|}\right\rangle .
$$

In the following discussion, we will call this $\mathbb{Z}_{M}$ rotation $\hat{g}^{m}$ as an $m$-twist, where $m \in \mathbb{Z} \bmod M \cdot \boldsymbol{x}=(x, \bar{x})$ are complex coordinates for the perpendicular directions, $x=x_{\perp}+i \tau, \bar{x}=x_{\perp}-i \tau$.

By using the orbifold method, EE can be easily calculated for free theories [61]. In the case of a real scalar field theory, the free energy takes the following form,

$$
\begin{aligned}
F_{\text {free }}^{(M)} & =\frac{1}{2} \operatorname{Tr} \log \left[\hat{P}\left(-\square+m_{0}^{2}\right)\right] \\
& =\frac{1}{2} \int \frac{d^{2} \boldsymbol{k}}{(2 \pi)^{2}} \frac{d^{d-1} k_{\|}}{(2 \pi)^{d-1}} \log \left(k^{2}+m_{0}^{2}\right)\left\langle\boldsymbol{k}, k_{\|}|\hat{P}| \boldsymbol{k}, k_{\|}\right\rangle
\end{aligned}
$$

The trace of $\hat{P}$ is computed as follows. The diagonal matrix element of $\hat{g}^{m}$ is given by

$$
\begin{aligned}
\left\langle\boldsymbol{k}, k_{\|}\left|\hat{g}^{m}\right| \boldsymbol{k}, k_{\|}\right\rangle & =\left\langle k_{\|} \mid k_{\|}\right\rangle\left\langle k, \bar{k}\left|\hat{g}^{m}\right| k, \bar{k}\right\rangle \\
& =(2 \pi)^{2} V_{d-1} \delta\left(\omega^{m} k-k\right) \delta\left(\omega^{-m} \bar{k}-\bar{k}\right),
\end{aligned}
$$

where $\quad V_{d-1}=(2 \pi)^{d-1} \delta^{d-1}\left(k_{\|}-k_{\|}\right) \quad$ is a transverse $(d-1)$-dimensional volume and $\omega=e^{2 \pi i / M}$. For $m \neq 0$, this becomes

$$
\left\langle\boldsymbol{k}, k_{\|}\left|\hat{g}^{m}\right| \boldsymbol{k}, k_{\|}\right\rangle=(2 \pi)^{2} V_{d-1} \delta^{2}(\boldsymbol{k}) \frac{1}{\omega^{m}-1} \frac{1}{\omega^{-m}-1} .
$$

For $m \neq 0$, it is proportional to $V_{d-1}$, the area of the boundary $\partial A$. On the other hand, for $m=0$, it is proportional to $V_{d-1} \times \delta^{2}(\mathbf{0}) \propto V_{d+1}$, the volume of the whole region of the path integral. From this, we see that twisting a propagator with $m \neq 0$ constrains the normal components of the momentum zero, $\boldsymbol{k}=0$. In Sec. IV A, we will have a slightly different interpretation of the twisted propagator.

The summation over $m$ from 1 to $M-1$ can be performed as follows. Given a holomorphic function $f(z)$, its summation is given by

$$
\sum_{m=1}^{M-1} f\left(\omega^{m}\right)=\oint_{C} \frac{d z}{2 \pi i} p(z) f(z),
$$

where

$$
p(z) \equiv \frac{M z^{M-1}}{z^{M}-1}-\frac{1}{z-1}
$$

has simple poles at $\omega^{m}=e^{2 \pi m i / M}$ with $m=1, \ldots, M-1$. The integration contour $C$ is chosen to surround these poles [64] (Fig. 3). Then the summation of Eq. (8) is calculated as 


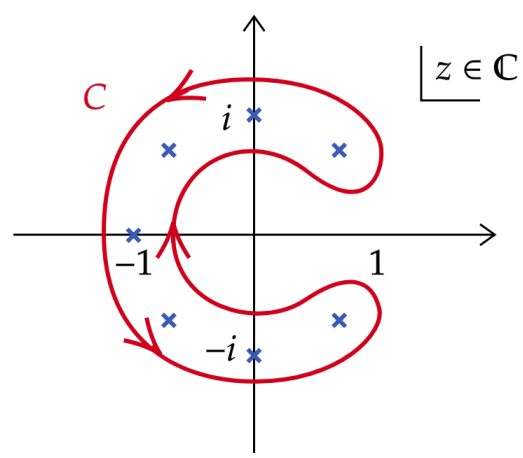

FIG. 3. The contour $C$ (red curve) and simple poles of $p(z)$ (blue cross marks) in Eq. (9) $(M=8$, as an example).

$\sum_{m=1}^{M-1} \frac{1}{\omega^{m}-1} \frac{1}{\omega^{-m}-1}=\oint_{C_{1}} \frac{d z}{2 \pi i} \frac{z p(z)}{(z-1)^{2}}=\frac{M^{2}-1}{12}$,

where $C_{1}$ is a counterclockwise circle around $z=1$. It is written as a compact form,

$$
\sum_{m=1}^{M-1} \frac{1}{\sin ^{2}\left(\frac{\pi m}{M}\right)}=\frac{M^{2}-1}{3} .
$$

Plugging Eq. (11) into Eq. (6), we obtain

$$
\begin{aligned}
F_{\text {free }}^{(M)}= & \frac{1}{2 M} \int \frac{d^{2} \boldsymbol{k} d^{d-1} k_{\|}}{(2 \pi)^{d-1}} \log \left(k^{2}+m_{0}^{2}\right) \\
& \times\left(\frac{V_{d+1}}{(2 \pi)^{2}}+V_{d-1} \frac{M^{2}-1}{12} \delta^{2}(\boldsymbol{k})\right) .
\end{aligned}
$$

The first term proportional to $V_{d+1}$ vanishes in the calculation of EE in Eq. (3). We will see later that such property generally holds even in presence of interactions. On the other hand, the second term is proportional to the area $V_{d-1}$ survives in Eq. (3) due to an additional $M$-dependence of $M^{2}-1$. Consequently, EE for a free scalar theory is given by

$$
S_{1 \text {-loop }}=-\frac{V_{d-1}}{12} \int^{1 / \epsilon} \frac{d^{d-1} k_{\|}}{(2 \pi)^{d-1}} \log \left[\left(k_{\|}^{2}+m_{0}^{2}\right) \epsilon^{2}\right] .
$$

Here we have introduced a UV cutoff $\epsilon$, which is naturally identified as a lattice spacing for a lattice system. Note that the EE decreases as the mass increases.

The calculation for the scalar theory can be easily generalized to bosonic higher spin theories [62]. In this case, a state is parametrized by $\left|\boldsymbol{x}, x_{\|} ; s\right\rangle$, where $s$ is a spin of $S O(2)$ rotation. Then the action of the two-dimensional rotation $\hat{g}$ is given by

$$
\hat{g}\left|x, \bar{x}, x_{\|} ; s\right\rangle=e^{2 \pi s i / M}\left|e^{2 \pi i / M} x, e^{-2 \pi i / M} \bar{x}, x_{\|} ; s\right\rangle
$$

and the sum over $m \neq 0$ in Eq. (12) is replaced by
$\sum_{m=1}^{M-1} \frac{\cos \left(\frac{2 \pi m s}{M}\right)}{\sin ^{2}\left(\frac{\pi m}{M}\right)}=\frac{1}{3}\left[M^{2}-1+6 M^{2}\left(\{s / M\}^{2}-\{s / M\}\right)\right]$,

where $\{x\}$ is a fractional part of $x$. For fermionic generalizations, we need special care since $2 \pi$ rotation gives an extra minus sign, $\hat{g}^{M}=-1$, and it cannot be regarded as $\mathbb{Z}_{M}$ orbifold. To overcome this difficulty, the authors in [62] take an odd $M$ and consider $\hat{g}^{2}$ as the generator of $\mathbb{Z}_{M}$ orbifold on a double cover of the Riemann surface. Another subtlety in higher spin generalizations in analytical continuation of $M$ since Eq. (16) contains a nonanalytic function, $\{s / M\}$, and we need to constrain the value of $s$ within $[-M, M]$ for fermions or $[0,2 M]$ for bosons. Thus the calculation of EE for higher spins than 3/2 may have subtlety in the orbifold method. For more details, see [62].

\section{ORBIFOLD METHOD IN AN INTERACTING FIELD THEORY}

In this section, we apply the orbifold method to interacting field theories and calculate the free energy of the $\mathbb{Z}_{M}$ orbifold. Each propagator in a Feynman diagram is projected by the projection operator $\hat{P}$ and thus we need to sum all the twists, $m \in \mathbf{Z}_{M}$ in each propagator. But there are redundancies in the summation, associated with $\mathbb{Z}_{M}$ rotations at each vertex in Feynman diagrams, and it is not so trivial to extract relevant terms that contribute to EE in Eq. (3). We first show that such redundancies can be systematically treated by performing the summation in the framework of the $\mathbb{Z}_{M}$ gauge theory on Feynman diagrams. Namely, assign $\mathbb{Z}_{M}$ twists on each link (i.e., on a propagator) and define $\mathbb{Z}_{M}$ gauge transformations on each vertex, and take a summation over all the twists modulo $\mathbb{Z}_{M}$ gauge transformations. Then, a gauge-invariant configuration of twists is characterized by a set of fluxes of twists on each plaquette of each Feynman diagram. Within this framework, we can easily prove the area law of EE.

\section{A. Setup}

Consider, for simplicity, a $\phi^{4}$ scalar field theory on a $\mathbb{Z}_{M}$ orbifold without a nonminimal coupling to the curvature. The action is given by

$$
\begin{aligned}
I= & \int_{\mathbb{R}^{2}} \frac{d^{2} x}{M} \int_{\mathbb{R}^{d-1}} d^{d-1} x_{\|}\left[\frac{1}{2}(\hat{P} \phi)\left(-\square+m_{0}^{2}\right)(\hat{P} \phi)\right. \\
& \left.+\frac{\lambda}{4}(\hat{P}(\phi))^{4}\right]
\end{aligned}
$$

in terms of a field $\phi(x)$ in flat space $\mathbb{R}^{2} \times \mathbb{R}^{d-1}$ but with the projection operator, where 

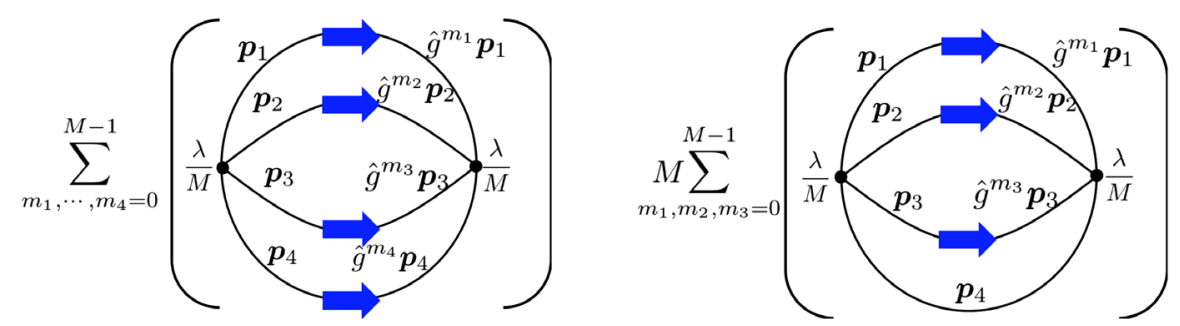

FIG. 4. Two equivalent configurations of twists in the three-loop diagram. Blue arrows denote twisted momenta with a twist $m_{i}$. The bottom propagator in the right is made untwisted by a $\mathbb{Z}_{M}$ rotation at a vertex.

$$
\hat{P} \phi\left(x, x_{\|}\right):=\frac{1}{M} \sum_{m=0}^{M-1} \phi\left(\hat{g}^{m} \boldsymbol{x}, x_{\|}\right)
$$

From the action Eq. (17), the inverse propagator of the orbifold theory in flat space is given by

$$
\hat{G}_{0}^{-1(M)}=\frac{\hat{P} \hat{G}_{0}^{-1} \hat{P}}{M}=\frac{\hat{P}\left(-\square+m_{0}^{2}\right) \hat{P}}{M}
$$

and the propagator, which satisfies $\hat{G}_{0}^{-1(M)} \hat{G}_{0}^{(M)}=\hat{P}$, is then written as

$$
G_{0}^{(M)}(x, y)=M\left\langle x\left|\left(\hat{P} \hat{G}_{0} \hat{P}\right)\right| y\right\rangle=\sum_{m=0}^{M-1} G_{0}\left(\hat{g}^{m} x, y\right),
$$

where

$$
\begin{aligned}
G_{0}\left(\hat{g}^{m} x, y\right) & =\int \frac{d^{d+1} p}{(2 \pi)^{d+1}} \frac{e^{i p \cdot\left(\hat{g}^{m} x-y\right)}}{p^{2}+m_{0}^{2}} \\
& =\int \frac{d^{2} \boldsymbol{p}}{(2 \pi)^{2}} \frac{d^{d+1} p_{\|}}{(2 \pi)^{d+1}} \frac{e^{i p \cdot\left(\hat{g}^{m} x-y\right)+i p_{\|} \cdot\left(x_{\|}-y_{\|}\right)}}{p^{2}+m_{0}^{2}} .
\end{aligned}
$$

The $\mathbb{Z}_{M}$ rotation on $y$ has been eliminated since a projection operator $\hat{P}$ commutes with $\hat{G}_{0}$. From the identity $\boldsymbol{p} \cdot \hat{g}^{m} \boldsymbol{x}=\hat{g}^{-m} \boldsymbol{p} \cdot \boldsymbol{x}$, we see that the flow-in momentum from the propagator at a vertex $x$ is given by the twisted momentum $\left(\hat{g}^{-m} \boldsymbol{p}, p_{\|}\right)$. In the momentum space representation, the propagator is written as

$$
\begin{aligned}
& \left\langle\boldsymbol{p}, p_{\|}\left|G^{(M)}\right| \boldsymbol{q}, q_{\|}\right\rangle \\
& =\sum_{m=0}^{M-1} \frac{1}{p^{2}+m_{0}^{2}}(2 \pi)^{d+1} \delta^{2}\left(\hat{g}^{m} \boldsymbol{p}-\boldsymbol{q}\right) \delta^{d-1}\left(p_{\|}-q_{\|}\right)
\end{aligned}
$$

with $-m$ redefined as $m$. The interaction vertex in the Euclidean $\phi^{4}$-theory read off from the action Eq. (17) is

$$
-3 ! \frac{\lambda}{M}
$$

The $x$ integration gives the ordinary momentum conserving delta functions

$$
\delta^{2}\left(\sum_{i} \hat{g}^{m_{i}} \boldsymbol{p}_{i}\right) \delta^{d-1}\left(\sum_{i} p_{i \|}\right)
$$

with a twisted flow-in momentum.

\section{B. Area law of EE in orbifold method}

We first show the area law of EE, i.e., $S_{A} \propto V_{d-1}$ (for a review of this property see [65] for example). There are two factors responsible for the area law: an overall dependence of the free energy on $M$, and the nontrivial argument in the momentum-conserving delta function in Eq. (24). Consider a Feynman diagram with $N_{V}$ vertices, $N_{P}$ propagators, and $L$ loops. Each vertex has a factor $1 / M$, and we may think naively that we have an overall factor of $(1 / M)^{N_{V}}$ in the free energy. However, this is not correct for the following reason. While we have $N_{P}$ summations over the twists, some of the $\mathbb{Z}_{M}$ summations are trivial due to the $\mathbb{Z}_{M}$ invariance of each vertex

$$
\delta^{2}\left(\sum_{i} \boldsymbol{p}_{i}\right)=\delta^{2}\left(\hat{g}^{n} \sum_{i} \boldsymbol{p}_{i}\right) \quad\left(\hat{g}^{n}: \text { an arbitrary twist }\right) .
$$

and give additional overall $M$ factors. Diagrammatically, we can untwist a part of twisted momenta on the propagators, i.e., eliminate some of the twists by using the above invariance of the $\delta$-function. Then the summations over the corresponding twists give overall factors of $M$. See Fig. 4 for an example. However, not all the $\mathbb{Z}_{M}$ rotations at $N_{V}$ vertices are independent. When we eliminate the twists of momenta by delta functions at vertices, we necessarily encounter the last delta function with no room for untwisting,

$$
\delta^{2}\left(\sum_{l=1}^{L}\left(1-\hat{g}^{m_{l}}\right) \boldsymbol{p}_{l}\right) \delta^{d-1}(0) .
$$

Here $p_{l}$ 's are all independent, nothing but the loop momenta. The number of the residual twists, which cannot be untwisted anymore, consistently coincides with $L$ because we can untwist $N_{V}-1$ out of $N_{P}$ twists. $^{1}$ As a result, the trivial

\footnotetext{
${ }^{1}$ Note that $N_{V}-N_{P}+L=1$.
} 
sums give an overall factor $M^{N_{V}-1}$. After all, the overall $M$-dependent factor of a general bubble diagram is given by

$$
\left(\frac{1}{M}\right)^{N_{V}} \times M^{N_{V}-1}=\frac{1}{M}
$$

As for the second point, we need to look into the argument of the delta functions. The $(d-1)$-dimensional delta function in Eq. (26) yields the $(d-1)$-dimensional volume: $\delta^{d-1}(0) \propto V_{d-1}$. Consequently, the diagram itself is formally expressed as

$\sum_{\{m\}} \frac{1}{M} V_{d-1} \int \prod_{l=1}^{L}\left[\frac{d^{2} \boldsymbol{p}_{l}}{(2 \pi)^{2}}\right] I(\{\boldsymbol{p}\} ;\{m\}) \delta^{2}\left(\sum_{l=1}^{L}\left(1-\hat{g}^{m_{l}}\right) \boldsymbol{p}_{l}\right)$,

where $I(\{\boldsymbol{p}\} ;\{m\})$ is a function of momenta and twists. We have to sum up diagrams over various configurations of the twists. First, note that the two-dimensional delta function generically has a nontrivial argument. They are proportional to $V_{d-1}$, the area of the boundary. The only exception is the configuration with trivial twists $m_{1}=\cdots=m_{L}=0$. It is identical to the corresponding diagram in flat space, where the diagram has a factor $\delta^{2}(\mathbf{0})$. It is then proportional to $V_{d-1} \times V_{2}$, the volume of the bulk.

The above statement holds for every bubble diagram. It leads to the following formal expression of the free energy:

$$
F^{(M)}=\frac{1}{M} F_{\text {flat }}+\tilde{F}_{\text {twisted }}^{(M)}, \quad \tilde{F}_{\text {twisted }}^{(M)}=V_{d-1} \frac{f(M)}{M},
$$

where $F_{\text {flat }}$ is the free energy of the $M=1$ field theory and $f(M)$ is an intensive quantity with a nontrivial dependence on $M$. Now we can readily check the area law from the above expression just in the same manner as in the free theory case. Although the first term is proportional to the volume of the bulk, it does not contribute to EE by the formula Eq. (3). In contrast, $\tilde{F}_{\text {twisted }}^{(M)}$ is proportional to the area of the boundary, and it does contribute to EE due to the $M$-dependence of $f(M)$. This completes the proof of the area law to all orders. The proof applies to any locally interacting theories. ${ }^{2}$ As a comment, it is also interesting to see a deviation from the area law by applying our formalism to theories exhibiting the volume law (e.g., manifestly nonlocal theories [66] and ones with nonlocal properties in some limit, Lifshitz field theories [67-69], for example) or the logarithmic violation to the area law (e.g., (non-)Fermi liquid theories [70]).

\footnotetext{
${ }^{2}$ We have additional phase factors due to spins, but they do not alter the result.
}

\section{C. $\mathbb{Z}_{M}$ gauge theory on Feynman diagrams}

The above statement is based on the idea that we can eliminate the redundant twists by using the invariance of the vertices under $\mathbb{Z}_{M}$ rotations. This procedure reminds us of gauge fixing in an ordinary gauge theory. In the following, we will show that this analogy works well in the investigation and that we can extract independent twists in a covariant manner. We call this methodology $\mathbb{Z}_{M}$ gauge theory on Feynman diagrams.

On a $\mathbb{Z}_{M}$ orbifold, each propagator in a Feynman diagram is twisted as in Eq. (20) and it is oriented by the twist number $m \in \mathbb{Z} \bmod M$. As an example, see Fig. 5. Four propagators with twist numbers $n_{1}, \ldots, n_{4}$ form one single loop. When we rotate the coordinates at a vertex $x$ by $2 \pi l / M, n_{1}$ and $n_{2}$ are shifted by $l$ and $-l$, respectively. Therefore, the sum of twists around a plaquette $m=\sum_{i} n_{i}$, which we sometimes call a flux, is invariant under $\mathbb{Z}_{M}$ rotations at vertices. It follows that for a given diagram such as the right figure of Fig. 5, independent configurations of twists are characterized by twist numbers of plaquettes. This is why the number of independent twists coincides with the number of loops L. For convenience, we assign the complement twist number to the outer circle $\left(m_{6}=-\sum_{l=1}^{5} m_{l}\right.$ in the right figure of Fig. 5), while it is not an independent twist. In the following, we omit writing such a twist assigned in an outer circle.

This prescription can be understood clearly when we interpret the twists as a kind of gauge fields. Regarding vertices in a Feynman diagram as topologically connected "sites" on a lattice. Since a twist on a propagator is defined between the two vertices, it can be seen as a link variable associated with the relative phase of the vertices. Then, the $\mathbb{Z}_{M}$ rotation on each vertex is interpreted as a local change of the phase. It is nothing but a gauge transformation, but the angle is restricted to $(2 \pi m / M)$ with $m=0, \ldots, M-1$.
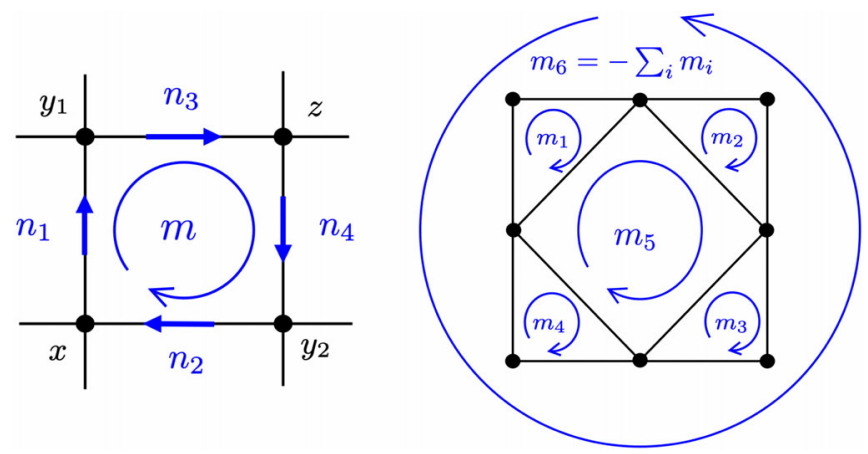

FIG. 5. $\mathbb{Z}_{M}$ fluxes on Feynman diagrams: The left figure shows twists on links (i.e., propagators) $\left\{n_{i}\right\}$ and the flux on the plaquette, which is given by a sum of twists around the plaquette $m=\sum_{i} n_{i}$ and invariant under local $\mathbb{Z}_{M}$ gauge transformations on vertices. A configuration of $\mathbb{Z}_{M}$-invariant twists is given by the fluxes on plaquettes (right). 
As a result, it is understood as $\mathbb{Z}_{M^{-} \text {gauge theory on }}$ Feynman diagrams. A flux in a plaquette, namely a sum of twists around the plaquette, is invariant under $\mathbb{Z}_{M}$ rotations at vertices and characterizes distinct configurations. Thus it is a counterpart of the Wilson loop, a gaugeinvariant object in a gauge theory. A flux of twists is defined as a sum of the twists of propagators in a counterclockwise direction along a plaquette. The flux is, of course, defined modulo $M$; i.e., $-m$ flux is equivalent to $M-m$ flux.

The procedure to calculate EE is straightforward: perform momentum integrations of each bubble Feynman diagram with a fixed configuration of twists, i.e., fluxes $\left\{m_{l}\right\}$ for plaquettes, and sum up them over all the twist configurations of $m$ 's. Then sum all the bubble diagrams as usual to obtain the free energy. Since the configuration of trivial twists does not contribute to $\mathrm{EE}$ as discussed in the previous section, we are interested in a configuration of twists, in which some of them are nonvanishing. An evaluation of Feynman diagrams with nonvanishing twists is in principle straightforward but very involved since momenta are twisted. Thus our strategy is, instead of considering general configurations of twists, to focus on dominant contributions to EE. In particular, in the following sections, we consider two specific types of configurations, giving contributions from twisted propagators and those from twisted vertices. We discuss in Sec. VII why they will give dominant contributions to $\mathrm{EE}$ and how the rest of contributions are incorporated in the Wilsonian renormalization picture. ${ }^{3}$

\section{PROPAGATOR CONTRIBUTIONS TO EE}

Among various configurations of twists, we first focus on the configurations that a single propagator is twisted. Consider a configuration where two plaquettes with a nonvanishing flux of twists share a propagator and their fluxes are given by $m$ and $-m$ respectively. For such a diagram, both of the fluxes can be attributed to the $m$-twist of the shared propagator (Fig. 6) and we can interpret such a flux configuration as a twist of the propagator. The contributions to $\mathrm{EE}$ from such a class of diagrams are then understood as two-point function contributions. We will investigate it both in the perturbative and nonperturbative approaches.

One might suspect whether a configuration of fluxes like Fig. 6 has a one-to-one correspondence to a configuration of a twist of the propagator in general diagrams. Indeed, we need careful treatment for particular diagrams. Consider a

\footnotetext{
${ }^{3}$ Although in Sec. IV and V, flux configurations that cannot be attributed to a single twist of either a propagator or a vertex remain uncalculated, we can address this issue via the Wilsonian renormalization group, which will be discussed in Sec. VII.
}

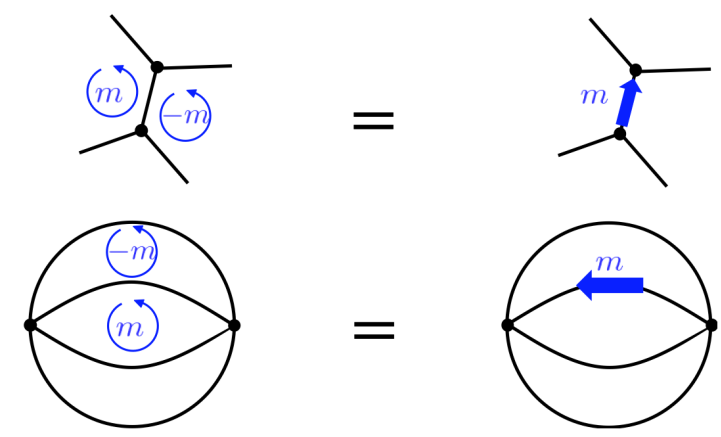

FIG. 6. If fluxes of plaquettes straddling a shared propagator are given by $m$ and $-m$, such a configuration is interpreted as a twist of the shared propagator. The upper figures show a relevant part with the twisted propagator in general diagrams.

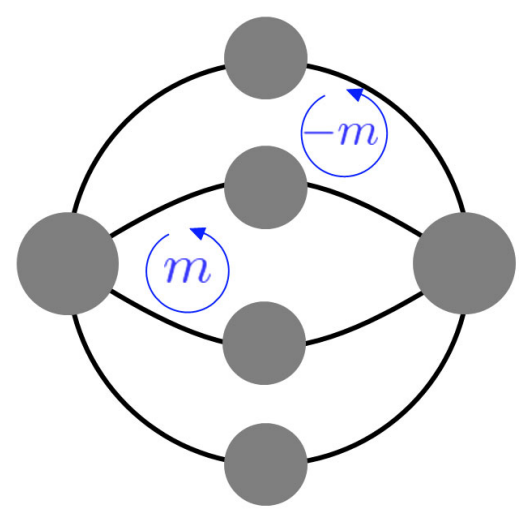

FIG. 7. An example of a configuration of fluxes $(m,-m)$ that has multiple interpretations in terms a twist of a bare propagator. The gray blobs represent 1PI subdiagrams. This configuration can be interpreted as a twist of either one of the two shared (bare) propagators, but not both.

diagram like Fig. 7 where two plaquettes with nonzero fluxes meet at two or more propagators. In this case, the configuration of fluxes $(m,-m)$ corresponds to a twist of either propagator, but not to both. This example shows that such a configuration of fluxes can be interpreted as a twist of the full propagator. In the following, we investigate propagator contributions to $\mathrm{EE}$ in more detail.

\section{A. Twisted propagator as a pinned propagator at the boundary}

Before proceeding to an investigation of individual diagrams, we address a concrete interpretation of a twisted propagator in order to get a physical intuition for twisting. We demonstrate below that a twisted propagator is pinned at the boundary. For this purpose, it is convenient to introduce the center-of-mass and relative coordinates: $X=(x+y) / 2, \quad r=x-y$. A twisted propagator with $m \neq 0$ in the position space is written as 


$$
\begin{aligned}
G_{0}\left(\hat{g}^{m} x-y\right)= & G_{0}\left(\hat{g}^{m / 2} \boldsymbol{x}-\hat{g}^{-m / 2} \boldsymbol{y} ; r_{\|}\right) \\
= & G_{0}\left(\cos \theta_{m} \boldsymbol{r}+2 \sin \theta_{m}(\epsilon \boldsymbol{X}) ; r_{\|}\right) \\
= & e^{\cot \theta_{m} \hat{R}_{X} / 2} G_{0}\left(2 \sin \theta_{m} \boldsymbol{X} ; r_{\|}\right) \\
= & \frac{e^{\cot \theta_{m} \hat{R}_{X} / 2}}{4 \sin ^{2} \theta_{m}} \int \frac{d^{d-1} k_{\|}}{(2 \pi)^{d-1}} \\
& \times \frac{e^{i k_{\|} \cdot r_{\|}}}{\left(-\partial_{X}^{2} / 4 \sin ^{2} \theta_{m}\right)+M_{k_{\|}}^{2}} \delta^{2}(\boldsymbol{X}),
\end{aligned}
$$

where

$$
\begin{aligned}
{[\epsilon \boldsymbol{X}]_{i}=} & \sum_{j} \epsilon_{i j} X_{j} \\
& \left(\epsilon_{i j}: \text { the two-dimensional Levi-Civita symbol }\right), \\
\hat{R}_{\boldsymbol{X}} & =\boldsymbol{r} \cdot\left(\epsilon \partial_{\boldsymbol{X}}\right), \quad \theta_{m}=\frac{m \pi}{M}, \quad M_{k_{\|}}^{2}=k_{\|}^{2}+m_{0}^{2} .
\end{aligned}
$$

Equation (30) can be written in a derivative expansion on the delta function with respect to $\partial_{X}^{2} / M_{k_{\|}}^{2}$. When we consider a diagram with a single twist $m$ on a propagator, it is formally written as

$$
\int d^{d+1} x d^{d+1} y G_{0}\left(\hat{g}^{m} x-y\right) F(r), \text { where } r=x-y .
$$

The integrand other than the twisted propagator only depends on $r$ due to the translational invariance. With Eq. (30) and the partial integration, we can drop all the $\partial_{\boldsymbol{X}}$ in the expression. Therefore, in this case, we can replace the propagator in the diagram as

$$
\begin{aligned}
G_{0}\left(\hat{g}^{m} x-y\right) & \rightarrow \frac{1}{4 \sin ^{2} \theta_{m}} \int \frac{d^{d-1} k_{\|}}{(2 \pi)^{d-1}} \frac{e^{i k_{\|} \cdot r_{\|}}}{M_{k_{\|}}^{2}} \delta^{2}(\boldsymbol{X}) \\
& =\frac{1}{4 \sin ^{2} \theta_{m}} G_{0}^{\mathrm{bdry}}\left(r_{\|}\right) \delta^{2}(\boldsymbol{X}) .
\end{aligned}
$$

$G_{0}^{\text {bdry }}$ is an ordinary propagator but its propagation is restricted only to the directions parallel to the boundary. Now the physical meaning of Eq. (34) is clear. Since the boundary of the subregion rests at the origin of the orbifold, the midpoint $\boldsymbol{X}$ of the twisted propagator is constrained on the boundary. Note that the propagator itself is not trapped on the boundary since the relative coordinate $\boldsymbol{r}$ is not constrained at all. Rather, $\boldsymbol{r}$-dependence completely disappears from the twisted propagator. Hence, it can be seen as a "pinned" propagator with two loose ends on the twodimensional plane. This shows that the twisted propagator reflects a correlation between two points that are symmetrically distant from the boundary (Fig. 8). In this sense, we can identify contributions to EE from a single twisted propagator as the quantum correlation of two-point functions. In Eq. (8), we saw that the one-loop contribution to EE from a twisted propagator gives a nontrivial delta

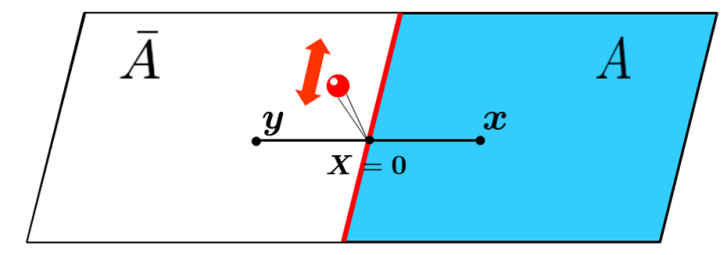

FIG. 8. An illustrative picture of a propagator pinned on the boundary. Its midpoint $\boldsymbol{X}=(\boldsymbol{x}+\boldsymbol{y}) / 2$ is constrained on the boundary $\partial A$ while two end points move freely.

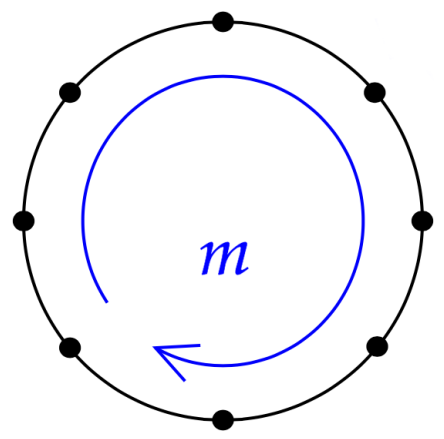

FIG. 9. There is a single twist for the 1-loop diagram consisting of a product of propagators.

function, $\delta^{2}(\boldsymbol{k})$, which is responsible for the area law of EE. Thus the above observation gives a different interpretation for the area law.

\section{B. Perturbative analysis}

We now investigate various diagrams containing a twisted propagator. Let us begin with 1-loop diagrams as shown in Fig. 9. In the perturbative approach, they are given by the Feynman diagrams with two-point vertices,

$$
\begin{aligned}
\operatorname{Tr} \log \left[\hat{P} G_{0}^{-1} \hat{P}\right] & =\int_{\epsilon^{2}} \frac{d s}{s} \operatorname{Tr} e^{-s G_{0}^{(M)} / M} \\
& =\int_{\epsilon^{2}} \frac{d s}{s} \sum_{n=0}^{\infty} \frac{(-s)^{n}}{n !} \operatorname{Tr}\left[\left(G_{0}^{(M)} \frac{1}{M}\right)^{n}\right] .
\end{aligned}
$$

These diagrams are exceptional in the sense that they are composed of a single chain of the propagators connected by the two-point vertices of $(1 / M){ }^{4}$ There is only one configuration with $m$-flux for the center plaquette. It can

\footnotetext{
${ }^{4}$ Reflecting the orbifold action in (17), the path integral measure is given by

$$
\int \mathcal{D}(\delta \phi) e^{-\frac{1}{2 M} \int d^{d+1} x(\delta \phi)^{2}}=1
$$

so that the 1-loop part of the free energy is given by $(1 / 2) \operatorname{Tr} \ln \left(\hat{P} G_{0}^{-1} \hat{P}\right)=(1 / 2) \operatorname{Tr} \ln \left(M\left(G_{0}^{(M)}\right)^{-1}\right) \quad$ rather than $(1 / 2) \operatorname{Tr} \ln \left(\left(G_{0}^{(M)}\right)^{-1}\right)$. See Eq. (19). This is responsible for the coefficient $(1 / M)$ of the two-point vertex in Eq. (35).
} 
be regarded as a twist of a single propagator among $n$ propagators in the expansion Eq. (35). This is what we have mentioned at the beginning of this section. From the viewpoint of operators, it corresponds to the idempotency of the projection: $\hat{P}^{2}=\hat{P}$.
In this case, it is convenient to take the momentum space representation of a twisted propagator, instead of the above position space interpretation. There is a single loop momentum $k$ and twisting results in a nonvanishing delta function of $\delta^{2}\left(\hat{g}^{m} \boldsymbol{k}-\boldsymbol{k}\right)$. The free energy is calculated as

$$
\begin{aligned}
\tilde{F}_{1-\text { loop }}^{(M)} & =\frac{1}{2} \operatorname{Tr} \ln \left(\hat{P} G_{0}^{-1} \hat{P}\right)=\frac{1}{2 M} \int_{\epsilon^{2}} \frac{d s}{s} \sum_{n=0}^{\infty} \frac{(-s)^{n}}{n !} \operatorname{Tr}\left[G_{0}^{n-1} G_{0}^{(M)}\right] \\
& =\frac{1}{2 M} \sum_{m=0}^{M-1} \int_{\epsilon^{2}} \frac{d s}{s} \sum_{n=0}^{\infty} \frac{(-s)^{n}}{n !} \int \frac{d^{2} \boldsymbol{k}}{(2 \pi)^{2}} \frac{d^{d-1} k_{\|}}{(2 \pi)^{d-1}}\left(\frac{1}{k^{2}+m_{0}^{2}}\right)^{n}(2 \pi)^{d+1} \delta^{2}\left(\hat{g}^{m} \boldsymbol{k}-\boldsymbol{k}\right) \delta^{d-1}(0) \\
& =\frac{1}{2 M} \int \frac{d^{2} \boldsymbol{k} d^{d-1} k_{\|}}{(2 \pi)^{d-1}} \log \left(k^{2}+m_{0}^{2}\right)\left(\frac{V_{d+1}}{(2 \pi)^{2}}+V_{d-1} \frac{M^{2}-1}{12} \delta^{2}(\boldsymbol{k})\right)
\end{aligned}
$$

and Eq. (13) is reproduced. Hence EE in the free theory is given by Eq. (14). In terms of the restricted propagator $G_{0}^{\text {bdry }}$ on the boundary, it is written as

$S_{1 \text {-loop }}=-\frac{V_{d-1}}{12} \int^{1 / \epsilon} \frac{d^{d-1} k_{\|}}{(2 \pi)^{d-1}} \ln \left[\left(\tilde{G}_{0}^{\text {bdry }}\left(k_{\|}\right)\right)^{-1} \epsilon^{2}\right]$,

where $\tilde{G}_{0}^{\text {bdry }}$ is the momentum space representation of $G_{0}^{\text {bdry }}$. $\left(\tilde{G}_{0}^{\text {bdry }}\left(k_{\|}\right)\right)^{-1}=k_{\|}^{2}+m_{0}^{2}$ is an effective squared mass on the two-dimensional plane with nonzero transverse momentum $k_{\|}$.

Next, we study contributions to EE from multiloops. Flux configurations of the 2-loop figure-eight diagram are characterized by twists $\left(m_{1}, m_{2}\right)$ on the two plaquettes. Its contribution to the free energy is given by

$\tilde{F}_{2 \text {-loop }}^{(M)}=\sum_{m_{1}, m_{2}} \frac{3 \lambda}{4 M} \int d^{d+1} x G_{0}\left(\hat{g}^{m_{1}} x, x\right) G_{0}\left(\hat{g}^{m_{2}} x, x\right)$.

Specific configurations of twists, $(m, 0)$ and $(0, m)$ with $m \neq 0$, correspond to a twist of each propagator (Fig. 10).

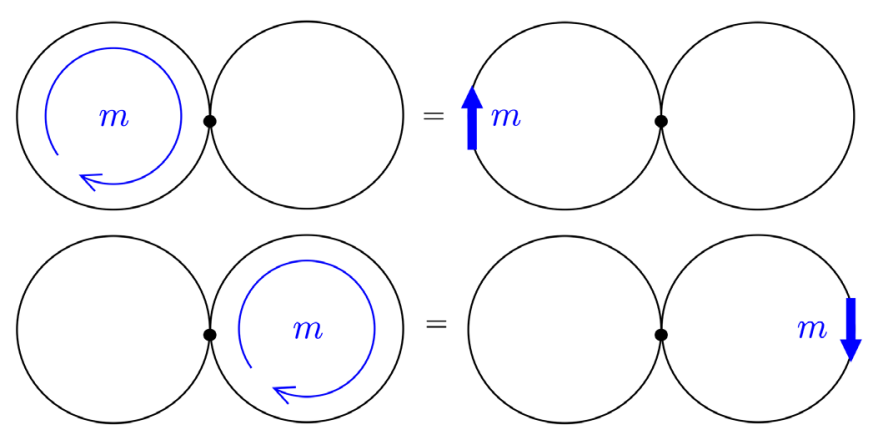

FIG. 10. 2-loop diagram with twists $\left(m_{1}, m_{2}\right)=(m, 0),(0, m)$ with $m \neq 0$ (left). They are interpreted as a twist of the corresponding propagators (right).
By using Eqs. (34) and (12), their contributions to the free energy and EE are computed respectively as

$$
\begin{aligned}
\tilde{F}_{2 \text {-loop,prop }}^{(M)}= & 2 \times \frac{3 \lambda}{4 M} \sum_{m=1}^{M-1} \int d^{d+1} x \frac{1}{4 \sin ^{2} \theta_{m}} G_{0}^{\text {bdry }}(0) \\
& \times \delta^{2}(\boldsymbol{x}) G_{0}(0) \\
= & V_{d-1} \frac{3 \lambda\left(M^{2}-1\right)}{24 M} G_{0}(0) G_{0}^{\mathrm{bdry}}(0), \\
S_{2 \text {-loop,prop }}= & -\frac{V_{d-1}}{12} G_{0}^{\mathrm{bdry}}(0)\left[3 \lambda G_{0}(0)\right] \\
= & -\frac{V_{d-1}}{12} \int \frac{d^{d-1} k_{\|}}{(2 \pi)^{d-1}} \tilde{G}_{0}^{\text {bdry }}\left(k_{\|}\right)\left[3 \lambda G_{0}(0)\right] .
\end{aligned}
$$

Note that the vertex contribution to EE is negative for the repulsive (positive $\lambda$ ) interaction. It is consistent with an expectation that the degrees of freedom must be reduced by introducing a positive $\lambda$ (otherwise the system becomes unstable) interaction.

Equation (41) indicates that this contribution to EE can be attributed to the mass renormalization to the 1-loop contribution of Eq. (38):

$$
\begin{aligned}
& S_{1 \text {-loop }}+S_{2 \text {-loop,prop }} \\
& \quad=-\frac{V_{d-1}}{12} \int^{1 / \epsilon} \frac{d^{d-1} k_{\|}}{(2 \pi)^{d-1}} \ln \left[\left(\tilde{G}_{1}^{\text {bdry }}\left(k_{\|}\right)\right)^{-1} \epsilon^{2}\right], \\
& \tilde{G}_{1}^{\text {bdry }}\left(k_{\|}\right)=\frac{1}{k_{\|}^{2}+m_{r 1}^{2}}, \quad m_{r 1}^{2}=m_{0}^{2}+3 \lambda G_{0}(0) .
\end{aligned}
$$

The above equalities hold up to $O\left(\lambda^{1}\right)$. This was also suggested in [37] to $O\left(\lambda^{1}\right)$.

When we compute higher-order contributions by explicit calculations, we observe that the propagator contributions are absorbed in the ordinary renormalization of the 
propagator order by order. This fact comes from the property explained in Fig. 7 that $(m,-m)$ type configurations of fluxes straddling many consecutive bare propagators will twist the corresponding single full propagator. It is not a trivial fact, but physically natural since EE is a measure of entanglement among microscopic degrees of freedom and should be related to the low-energy observables through renormalization. This observation motivates us to pursue the following analysis that $\mathrm{EE}$ (or at least its universal term) is expressed in terms of renormalized correlation functions in the 2PI formalism.

\section{Nonperturbative analysis in 2PI formalism}

In order to study a relationship between renormalization of propagators and EE more systematically, we employ the framework of the two-particle irreducible (2PI) formalism $[59,60]$. Combined with the orbifold analysis, we will confirm that the Gaussian contributions to EE are completely expressed in terms of the renormalized two-point function in the following.

The 2PI effective action is given by

$$
\Gamma[G]=\frac{1}{2} \operatorname{tr} \log G^{-1}+\frac{1}{2} \operatorname{tr}\left(G_{0}^{-1} G-1\right)+\Gamma_{2}[G],
$$

where $G$ is a full propagator, namely, a renormalized twopoint function. $\Gamma_{2}[G]$ is minus the sum of connected $2 \mathrm{PI}$ bubble diagrams which consist of the full propagators $G$ 's as internal lines. We assume that the one-point function vanishes: $\langle\phi\rangle=0$. In this formalism, $G$ is determined self-consistently by its equation of motion, called a gap equation:

$$
\frac{\delta \Gamma[G]}{\delta G}=0 \Leftrightarrow G^{-1}=G_{0}^{-1}+2 \frac{\delta \Gamma_{2}}{\delta G}[G] .
$$

With the solution to Eq. (45), $G=\bar{G}\left[G_{0}\right], \Gamma[\bar{G}]$ coincides with the 1PI free energy. Thus, what we need to evaluate is $\Gamma[\bar{G}]$ with a single full propagator being twisted.

In the 2PI analysis, since $G(x, y)$ itself is composed of propagators as internal loop corrections, we distinguish the following two types of twistings. The first type of twistings is denoted by $\delta_{m} G(x, y)$, which represents a variation of the internal structure induced by twisting. The second type is simply given by $G\left(\hat{g}^{m} x, y\right)$, which represents the twisting of the full propagator in the same way as previously. Namely, the projection operator $\hat{P}$ is acted from outside. We will show that the first type of twistings is canceled by the gap equation. Moreover, we will prove that there are further cancellations among 2PI diagrams and the second term of the 2PI effective action in Eq. (44). The gap equation is responsible for the cancellations, but special care is necessary for such diagrams in Fig. 7.
First let us see that twistings inside the full propagators are canceled and contributions from $\delta_{m} G(x, y)$ vanish. It is simply because of the gap equation;

$$
\begin{aligned}
\Gamma[\bar{G}]_{\text {prop,int }}= & \sum_{m=1}^{M-1} \int d^{d+1} x d^{d+1} y \\
& \times \frac{1}{2}\left(-\bar{G}^{-1}+G_{0}^{-1}+2 \frac{\delta \Gamma_{2}}{\delta G}[\bar{G}]\right)_{y x} \delta_{m} G(x, y) \\
= & 0 .
\end{aligned}
$$

Thus we can safely forget about the internal structure of the full propagator.

Next, we look at the twisting of the full propagator itself. As expected, most configurations with a single twisted propagator are canceled due to the gap equation, except for diagrams like Fig. 7 where a configuration of fluxes of $(m,-m)$ can be attributed to twisting one of the propagators straddled by the plaquettes. In the 2PI formalism, such diagrams are included only in the first term in Eq. (44) because all diagrams with such property are not 2PI (see Fig. 7) and not included in other terms. ${ }^{5}$ Then, we can separately consider contributions from the first term and those from the second and third term in Eq. (44).

The first term gives the same form of EE as in the free theory. A flux of twists is present in the center plaquette, which can be attributed to one of the propagators, but not to all. The situation is completely the same as in the 1-loop analysis in the previous section, and it results in the following contributions to EE,

$S_{\text {prop }, \text { ext }, 1}^{\text {PI }}=-\frac{V_{d-1}}{12} \int^{1 / \epsilon} \frac{d^{d-1} k_{\|}}{(2 \pi)^{d-1}} \log \left[\tilde{G}^{-1}\left(\mathbf{0} ; k_{\|}\right) \epsilon^{2}\right]$,

where $\tilde{G}\left(\boldsymbol{k} ; k_{\|}\right)$is the Fourier transform of $\bar{G}(x, y) . \tilde{G}\left(\mathbf{0} ; k_{\|}\right)$ is a renormalized counterpart of $\tilde{G}^{\text {bdry }}$. Note that, though $\tilde{G}_{0}^{\text {bdry }}\left(k_{\|}\right)$describes a propagation in a $(d-1)$-dimensional theory, the renormalization of $\tilde{G}\left(\mathbf{0} ; k_{\|}\right)$itself is performed in the $(d+1)$-dimensional space, as in Eq. (42).

As for the second and third terms in Eq. (44), their contributions to $\mathrm{EE}$ are given by

$$
\begin{aligned}
S_{\text {prop }, \text { ext }, 2+3}^{2 \text { PI }}= & \sum_{m=1}^{M-1} \int d^{d+1} x d^{d+1} y\left(\frac{1}{2} G_{0}^{-1}+\frac{\delta \Gamma_{2}}{\delta G}[\bar{G}]\right)_{y x} \\
& \times \bar{G}\left(\hat{g}^{m} x, y\right) \\
= & \sum_{m=1}^{M-1} \int d^{d+1} x d^{d+1} y\left(\frac{1}{2} \bar{G}^{-1}\right)_{y x} \bar{G}\left(\hat{g}^{m} x, y\right)
\end{aligned}
$$

\footnotetext{
${ }^{5}$ The second term is not $2 \mathrm{PI}$, but $G_{0}^{-1}$ is a local operator and it is sufficient to twist the propagator $G$ in the trace.
} 

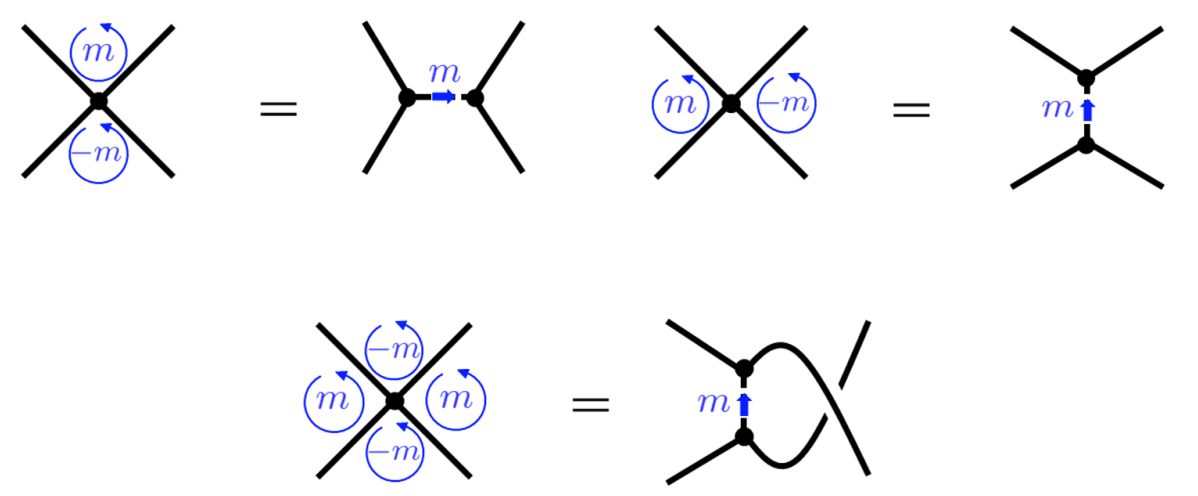

FIG. 11. Twisting a vertex: these three types of configurations can be attributed to a twist of a vertex. The dotted lines in the figures on the right-hand sides are delta functions to open the vertex. The twist of a vertex is interpreted as a twist of the dotted propagator. Each set of figures represent the three channels, $s$-channel (upper left figures), $t$-channel (upper right figures) and $u$-channel (lower figures) respectively.

Since the last expression is just a variation of unity, $S_{\text {prop }, \text { ext }, 2+3}^{2 \mathrm{PI}}$ is a trivial constant and can be dropped.

By combining Eqs. (47), (48), we obtain the contribution to EE from twisting a propagator in terms of the renormalized two-point function nonperturbatively:

$$
S_{\text {prop }}^{2 \mathrm{PI}}=-\frac{V_{d-1}}{12} \int^{1 / \epsilon} \frac{d^{d-1} k_{\|}}{(2 \pi)^{d-1}} \log \left[\tilde{G}^{-1}\left(\mathbf{0} ; k_{\|}\right) \epsilon^{2}\right]
$$

Previously we made a conjecture that the total propagator contribution to EE could be represented as renormalization of the propagator. The above argument completes the proof. The Gaussian contribution is all summarized in the above form. ${ }^{6}$ Note that it is consistent with the leading order result of perturbative calculations in [37,38].

Though we have shown the above result for the Gaussian contribution to EE in a specific model of the $\phi^{4}$ theory, a generalization to other theories is almost straightforward and the above result is completely general. See Sec. VI for further details.

\section{VERTEX CONTRIBUTIONS TO EE}

In the previous sections, we have successfully extracted the Gaussian part of EE completely. The rest contributions to $\mathrm{EE}$ are purely non-Gaussian. Investigations of nonGaussian contributions to EE are more involved since these contributions are hidden in various configurations of twists. However, some of them do have a simple interpretation as we show in this section.

\footnotetext{
${ }^{6}$ When we compare Eq. (49) to the ordinary perturbative calculation, since all the diagrams in Eq. (44) are written in terms of the full propagator $G$, we have to expand each diagram in the comparison. As a result, diagrams consisting of $G_{0}$ 's are included in all the three terms in Eq. (44) and the correct coefficients can be obtained by taking all these terms into account.
}

\section{A. Perturbative analysis}

Such configurations with a simple interpretation are given by a set of flux configurations that straddle a vertex instead of a propagator. Consider a diagram with twists given schematically in Fig. 11. In these configurations, plaquettes with nonvanishing fluxes of twists meet at a vertex, and there are three types of such configurations. We can interpret these configurations as a configuration of a single twisted vertex in the $s, t$, and $u$-channel respectively. This interpretation can be realized by "opening" the vertex with a delta function. For example, the four-point vertex can be rewritten as

$$
\frac{\lambda}{4} \int d^{d+1} x \phi(x)^{4}=\frac{\lambda}{4} \int d^{d+1} x d^{d+1} y \phi(x)^{2} \phi(y)^{2} \delta^{d+1}(x-y) .
$$

Then, we can understand a twisted vertex as an opened vertex with a twist on the separated two coordinates as

$$
\frac{\lambda}{4} \int d^{d+1} x d^{d+1} y \phi(x)^{2} \phi(y)^{2} \delta^{d+1}\left(\hat{g}^{m} x-y\right)
$$

The upper left, upper right, and lower figures in Fig. 11 correspond to the $s, t$, and $u$-channel openings of the vertex, respectively. As we have demonstrated for a single twisted propagator, we can replace the twisted delta function (to be exact, its two-dimensional part) in the diagram as

$$
\delta^{2}\left(\hat{g}^{m} \boldsymbol{x}-\boldsymbol{y}\right)=e^{\cot \theta_{n} \hat{R}_{X} / 2} \frac{\delta^{2}(\boldsymbol{X})}{4 \sin ^{2} \theta_{m}} \rightarrow \frac{\delta^{2}(\boldsymbol{X})}{4 \sin ^{2} \theta_{m}} .
$$

The twisted vertex is thus interpreted as a vertex symmetrically splitted with two loose ends and also with its center coordinate being fixed at the boundary.

Let us evaluate these vertex contributions up to the 3-loop level. The 2-loop vertex contributions stem from the figure-eight diagram with two types of configurations of 


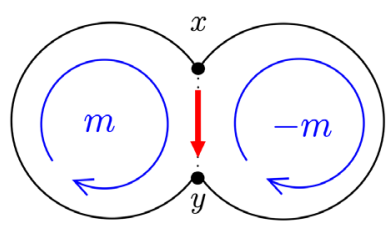

$m$

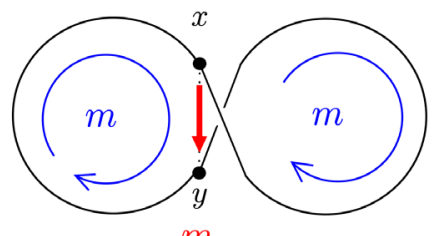

$m$

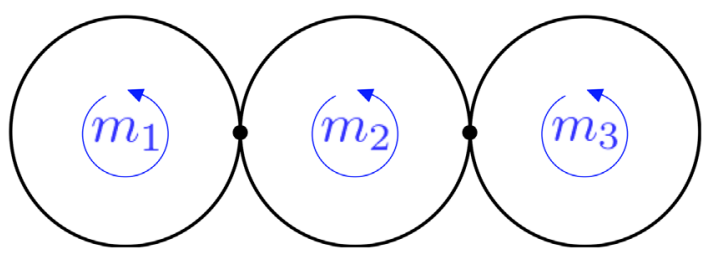

FIG. 12. 2-loop figure-eight diagrams with twists $\left(m_{1}, m_{2}\right)=$ $(m, \mp m)$. These flux configurations of twists can be interpreted as a twist of the 4-point vertex by decomposing it into two 3-point vertices.

twists, as shown in Fig. 12. Note that the configuration of the $s$-channel opening is absent in the figure-eight diagram because the vertex in the figure-eight diagram is surrounded by essentially three plaquettes, two circles, and one outer circle. Their contributions to the free energy and $\mathrm{EE}$ are calculated as

$$
\begin{aligned}
& \tilde{F}_{\text {2-loop.vert }}^{(M)}= 2 \times \sum_{m=1}^{M-1} \frac{3 \lambda}{4 M} \int d^{d+1} x d^{d+1} y G_{0}(x-y)^{2} \\
& \times \delta^{d-1}\left(x_{\|}-y_{\|}\right) \delta^{2}\left(\hat{g}^{m} \boldsymbol{x}-\boldsymbol{y}\right) \\
&= V_{d-1} \lambda \frac{M^{2}-1}{8 M} \int d^{2} \boldsymbol{r} G_{0}(\boldsymbol{r}, 0)^{2}, \\
& S_{2-\text { loop,vert }}=-V_{d-1} \frac{\lambda}{4} \int d^{2} \boldsymbol{r} G_{0}(\boldsymbol{r}, 0)^{2} .
\end{aligned}
$$

FIG. 13. A 3-loop diagram. Four types of flux configurations, $\left(m_{1}, m_{2}, m_{3}\right)=(m, \pm m, 0),(0, m, \pm m)$, can be interpreted as twisting vertices. Opening vertices are done in the same manner as in the 2-loop diagrams.

Note that in the real $\phi^{4}$ theory, different channels are indistinguishable and a summation of different channels give just an additional numerical factor in front. In the next section, we will consider an extended model in which a different channel gives a different type of contribution.

The 3-loop contributions come from two diagrams shown in Fig. 13 and Fig. 14. For a diagram illustrated in Fig. 13, the vertex contributions stem from the four configurations: $\left(m_{1}, m_{2}, m_{3}\right)=(m, \pm m, 0),(0, m, \pm m)$. We see them as $t$ - and $u$-channel opening of the two vertices. $s$-channels are absent because each vertex is surrounded by two plaquettes and one outer circle, not four independent ones. The contributions from these configurations to the free energy and $\mathrm{EE}$ are given by

$$
\begin{gathered}
\tilde{F}_{\text {3-loop,vert1 }}^{(M)}=4 \times\left(-\frac{9 \lambda^{2}}{4 M} \sum_{m=1}^{M-1} \int d^{d+1} x_{1} d^{d+1} x_{2} d^{d+1} y G_{0}\left(x_{1}-x_{2}\right) G_{0}\left(x_{1}-y\right) G_{0}\left(x_{2}-y\right) G_{0}(0) \delta^{d+1}\left(\hat{g}^{m} x_{1}-x_{2}\right)\right) \\
=-V_{d-1} \frac{3 \lambda^{2}\left(M^{2}-1\right)}{M} \int d^{2} \boldsymbol{x} d^{2} \boldsymbol{y} d^{d-1} r_{\|} G_{0}(2 \boldsymbol{x}, 0) G_{0}\left(\boldsymbol{x}-\boldsymbol{y}, r_{\|}\right) G_{0}\left(\boldsymbol{x}+\boldsymbol{y}, r_{\|}\right) G_{0}(\mathbf{0}, 0), \\
=-V_{d-1} \frac{3 \lambda^{2}\left(M^{2}-1\right)}{4 M} \int d^{2} \boldsymbol{r} d^{2} \boldsymbol{s} d^{d-1} r_{\|} G_{0}(\boldsymbol{r}, 0) G_{0}\left(\boldsymbol{r}-\boldsymbol{s}, r_{\|}\right) G_{0}\left(\boldsymbol{s}, r_{\|}\right) G_{0}(\mathbf{0}, 0), \\
S_{3 \text {-loop,vert1 }}=V_{d-1} \frac{3}{2} \lambda^{2} \int d^{2} \boldsymbol{r} d^{2} \boldsymbol{s} d^{d-1} r_{\|} G_{0}(\boldsymbol{r}, 0) G_{0}\left(\boldsymbol{r}-\boldsymbol{s}, r_{\|}\right) G_{0}\left(\boldsymbol{s}, r_{\|}\right) G_{0}(\mathbf{0}, 0), \\
=V_{d-1} \frac{3}{2} \lambda^{2} \int d^{d+1} r G_{0}(r) G_{0}(0)\left[\int d^{2} \boldsymbol{s} G_{0}(\boldsymbol{s}, 0) G_{0}\left(\boldsymbol{s}-\boldsymbol{r}, r_{\|}\right)\right] .
\end{gathered}
$$

Another 3-loop diagram is given by the leftmost diagram in Fig. 14. The following three types of configurations of twists correspond to twists of a vertex: $(0, m, 0)$, $(m, 0,-m)$, and $(m,-m, m)$. We can assign a flux of twist $0,-m$, and $-m$ on the outer circle of the plaquette respectively. They are equivalent to the $t$-, $s$ - and $u$-channel opening of the vertex. In this diagram, we again face the problem of the failure of one-to-one correspondence in Fig. 7. There are two ways to attribute the flux configurations to twisting either an upper or lower vertex. These two attributions are not independent and we can only twist one of them. These three channels give the same contributions in the $\phi^{4}$ theory. Then, the corresponding 3-loop contributions from Fig. 14 are computed as 

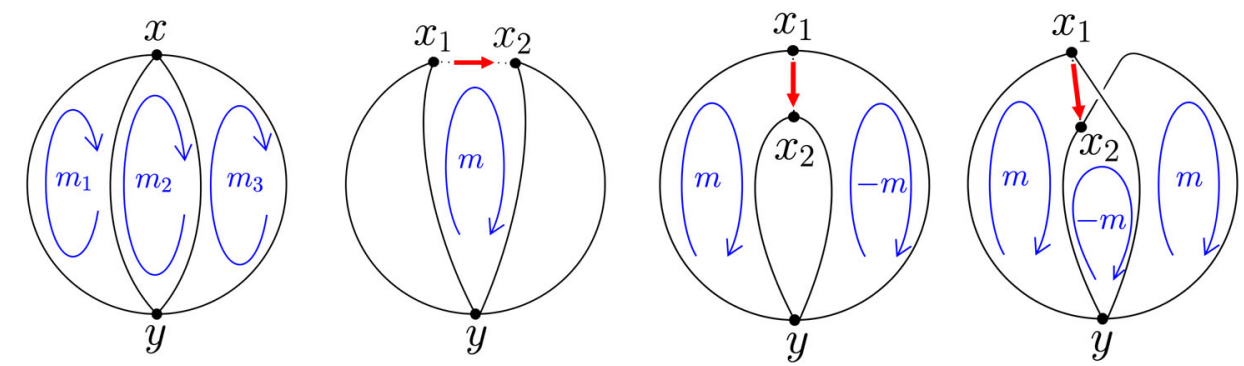

FIG. 14. Another 3-loop diagram with twists $\left(m_{1}, m_{2}, m_{3}\right)$ (leftmost). A particular configuration $(0, m, 0)$ corresponds to twisting a vertex, as well as $(m, 0,-m)$ and $(m,-m, m)$ (three diagrams on the right). They generate a twist in the delta function $\delta^{2}\left(\boldsymbol{x}_{1}-\boldsymbol{x}_{2}\right)$. We can also open the vertex at $\mathbf{y}$ instead of $\boldsymbol{x}$, and they have two different interpretations of twisting vertices, analogous to Fig. 7 . These vertex contributions should not be double-counted.

$$
\begin{aligned}
\tilde{F}_{3-\text { loop }, \text { vert2 }}^{(M)} & =3 \times\left(-\frac{3 \lambda^{2}}{4 M} \sum_{m=1}^{M-1} \int d^{d+1} x_{1} d^{d+1} x_{2} d^{d+1} y G_{0}\left(x_{1}-y\right)^{2} G_{0}\left(x_{2}-y\right)^{2} \delta^{d+1}\left(\hat{g}^{m} x_{1}-x_{2}\right)\right) \\
& =-V_{d-1} \frac{3 \lambda^{2}\left(M^{2}-1\right)}{4 M} \int d^{2} \boldsymbol{x} d^{2} \boldsymbol{y} d^{d-1} r_{\|} G_{0}\left(\boldsymbol{x}-\boldsymbol{y}, r_{\|}\right)^{2} G_{0}\left(\boldsymbol{x}+\boldsymbol{y}, r_{\|}\right)^{2}, \\
& =-V_{d-1} \frac{3 \lambda^{2}\left(M^{2}-1\right)}{16 M} \int d^{2} \boldsymbol{r} d^{2} \boldsymbol{s} d^{d-1} r_{\|} G_{0}\left(\boldsymbol{r}, r_{\|}\right)^{2} G_{0}\left(\boldsymbol{s}, r_{\|}\right)^{2},
\end{aligned}
$$

$$
\begin{aligned}
S_{3-\text { loop }, \text { vert2 }} & =V_{d-1} \frac{3 \lambda^{2}}{8} d^{2} \boldsymbol{r} d^{2} \boldsymbol{s} d^{d-1} r_{\|} G_{0}\left(\boldsymbol{r}, r_{\|}\right)^{2} G_{0}\left(\boldsymbol{s}, r_{\|}\right)^{2}, \\
& =V_{d-1} \frac{3 \lambda^{2}}{8} \int d^{d+1} r G_{0}(r)^{2}\left[\int d^{2} \boldsymbol{s} G_{0}\left(\boldsymbol{s}, r_{\|}\right)^{2}\right] .
\end{aligned}
$$

In contrast to the twisting of propagators, both of the contributions of Eqs. (56) and (58) essentially originate from the non-Gaussianity of the state. We also emphasize the importance of the covariant viewpoint as $\mathbb{Z}_{M}$ gauge theory on Feynman diagrams. If we take a special gauge and assign twists on specific links (propagators), we could not find out vertex contributions since they are hidden in the configurations with multiple twisted links.

While Eq. (56) can be interpreted as a contribution from the figure-eight diagram with the renormalized propagator Eq. (42), Eq. (58) cannot be absorbed into the renormalization of the propagator nor the vertex. The situation is different from the propagator contributions, nonetheless, it is consistent with the ordinary renormalization structure in another viewpoint. In the following, we will show that the above vertex contributions can be summarized as those from renormalized composite operators.

\section{B. Vertex contributions as correlations of composite operators}

In order to formulate the "opening of a vertex" more systematically, it is instructive to consider a model where opening each vertex leads to distinct $s-, t$ - and $u$-channels.
One of such models is described by two complex scalars, whose action is given by

$$
I=\int \frac{d^{d+1} x}{M}\left[\sum_{i=1}^{2} \bar{\phi}_{i}\left(-\square+m_{0}^{2}\right) \phi_{i}+\frac{\lambda}{4}\left(\bar{\phi}_{1} \phi_{1}\right)\left(\bar{\phi}_{2} \phi_{2}\right)\right] .
$$

Here and in the following, $\mathbb{Z}_{M}$ projections on fields are written implicitly. Each vertex contribution involves three configurations of twists as mentioned in Fig. 11. It is now almost clear that each twist of a vertex in $s, t$, and $u$ channels can be regarded as a twist of the propagator of the corresponding auxiliary field. With the auxiliary field, the action has a three-point interaction vertex and reproduces the original four-point one when integrated out.

Corresponding to the above three ways for the opening of vertices, we can rewrite the action Eq. (59) into the following three forms:

$$
\begin{aligned}
I_{s}= & \int \frac{d^{d+1} x}{M}\left[\sum_{i=1}^{2} \bar{\phi}_{i}\left(-\square+m_{0}^{2}\right) \phi_{i}+c_{1} c_{2}\right. \\
+ & \left.i \frac{\sqrt{\lambda}}{2} c_{1}\left(\bar{\phi}_{2} \phi_{2}\right)+i \frac{\sqrt{\lambda}}{2} c_{2}\left(\bar{\phi}_{1} \phi_{1}\right)\right], \\
I_{t}= & \int \frac{d^{d+1} x}{M}\left[\sum_{i=1}^{2} \bar{\phi}_{i}\left(-\square+m_{0}^{2}\right) \phi_{i}+\bar{d} d\right. \\
& \left.+i \frac{\sqrt{\lambda}}{2} \bar{d} \phi_{1} \phi_{2}+i \frac{\sqrt{\lambda}}{2} d \bar{\phi}_{1} \bar{\phi}_{2}\right],
\end{aligned}
$$




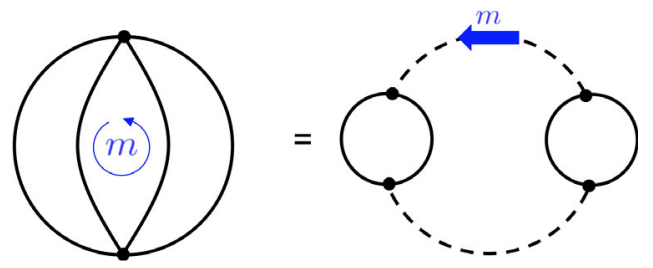

FIG. 15. The right non-2PI diagram is obtained by opening two vertices in the left in terms of the auxiliary fields. We can regard a flux of the center plaquette as a twist of either upper or lower vertex, but not both. In terms of the auxiliary field, it is nothing but the phenomena explained in Fig. 7.

$$
\begin{aligned}
I_{u}= & \int \frac{d^{d+1} x}{M}\left[\sum_{i=1}^{2} \bar{\phi}_{i}\left(-\square+m_{0}^{2}\right) \phi_{i}+\bar{d}^{\prime} d^{\prime}\right. \\
& \left.+i \frac{\sqrt{\lambda}}{2} \bar{d}^{\prime} \bar{\phi}_{1} \phi_{2}+i \frac{\sqrt{\lambda}}{2} d^{\prime} \bar{\phi}_{2} \phi_{1}\right] .
\end{aligned}
$$

We have introduced three pairs of auxiliary fields: real scalars $\left(c_{1}, c_{2}\right)$, and complex scalars $(d, \bar{d}),\left(d^{\prime}, \bar{d}^{\prime}\right){ }^{7}$ Of course, each of Eqs. (60)-(62) is equivalent to Eq. (59) after integrating the auxiliary fields out. Consequently, if we sum up the bubble diagrams from all three models, we will encounter an overcounting at the level of free energy. However, when we consider configurations of twists, there is a one-to-one correspondence between vertex contributions of three channels and propagator contributions of each auxiliary field in these three models. In this sense, as far as a single twist of vertices is concerned, the vertex contributions we consider can be regarded as the propagator contributions from these three auxiliary fields. As in Fig. 14, four-point vertex contributions to $\mathrm{EE}$ with the flux configurations, $(0, m, 0),(m, 0,-m)$, or $(m,-m, m)$, corresponds to a propagator contribution of the associated auxiliary fields given by Eqs. (60)-(62), respectively, for any bubble diagrams of the action Eq. (59).

Every vertex in the bubbles generated by Eq. (59) gets the contributions from the three channels. ${ }^{8}$ They coincide respectively with the contributions from a twisted propagator in the equivalent diagrams generated either by Eqs. (60).(62). Here we have the same problem of the one-to-one correspondence between fluxes of twists in the plaquettes and twists of vertices, as mentioned in the previous subsection (Fig. 14). In terms of the auxiliary fields, this problem is easily resolved by using the same logic as in the propagator contributions. 2PI diagrams do not have this kind of problem, and only 1-loop diagrams of the auxiliary

\footnotetext{
${ }^{7}$ The path integral contour for them should be chosen so that the partition function is convergent and thus the apparent violation of the reality or boundedness in the above actions does not produce pathology.

${ }^{8}$ Figure-eight diagram is an exception and there is no $s$-channel.
}

fields need care. See Fig. 15 as an example. As a result, the problem is translated into the same problem for the twisted propagator of the auxiliary field.

The above observation leads us to express EE in the 2PI formalism with the auxiliary fields. Although we cannot rewrite the action itself by using all the auxiliary fields simultaneously, the vertex contributions to the free energy and $\mathrm{EE}$ can be written as a sum of the contributions from these three. The result is given by ${ }^{9}$

$$
\begin{aligned}
S_{\mathrm{vert}}^{2 \mathrm{PI}}= & -\frac{V_{d-1}}{12}\left(\int^{1 / \epsilon} \frac{d^{d-1} k_{\|}}{(2 \pi)^{d-1}} \operatorname{tr} \log \left[\tilde{G}_{c}^{-1}\left(\mathbf{0} ; k_{\|}\right)\right]\right. \\
& +2 \int^{1 / \epsilon} \frac{d^{d-1} k_{\|}}{(2 \pi)^{d-1}} \log \left[\tilde{G}_{d}^{-1}\left(\mathbf{0} ; k_{\|}\right)\right] \\
& \left.+2 \int^{1 / \epsilon} \frac{d^{d-1} k_{\|}}{(2 \pi)^{d-1}} \log \left[\tilde{G}_{d^{\prime}}^{-1}\left(\mathbf{0} ; k_{\|}\right)\right]\right)
\end{aligned}
$$

Here, $\left(\tilde{G}_{c}\right)_{i j}, \tilde{G}_{d}$ and $\tilde{G}_{d^{\prime}}$ is the Fourier transformations of the two-point functions $\left\langle c_{i}(x) c_{j}(y)\right\rangle,\langle d(x) \bar{d}(y)\rangle$, and $\left\langle d^{\prime}(x) \bar{d}^{\prime}(y)\right\rangle$ and the first, second, and third terms in Eq. (63) represent the vertex contributions from the $s$-, $t$ - and $u$-channel openings, respectively. The coefficients " 2 " in the second and third lines come from the fact that $(d, \bar{d})$ and $\left(d^{\prime}, \bar{d}^{\prime}\right)$ are complex fields. $\left(c_{1}, c_{2}\right)$ are real fields, but its propagator is written as a $2 \times 2$ matrix and has two degrees of freedom. The $t r$ is the trace taken over this $2 \times 2$ matrix.

Equation (63) has a remarkable interpretation. Note that we can regard the auxiliary fields as degrees of freedom of composite operators:

$$
\begin{aligned}
& c_{1} \sim \bar{\phi}_{2} \phi_{2}, \quad c_{2} \sim \bar{\phi}_{1} \phi_{1}, \\
& d \sim \bar{\phi}_{1} \bar{\phi}_{2}, \quad \bar{d} \sim \phi_{1} \phi_{2}, \\
& d^{\prime} \sim \bar{\phi}_{2} \phi_{1}, \quad \bar{d}^{\prime} \sim \bar{\phi}_{1} \phi_{2} .
\end{aligned}
$$

They are justified in various ways, for instance, the vacuum expectation values of both sides coincide. From this viewpoint, Eq. (63) indicates that the vertex corrections are in fact understood as propagator contributions of the composite operators. From the actions Eqs. (60), (61), and (62), the propagators of auxiliary fields are written in terms of correlation functions of the above composite operators as

$$
\tilde{G}_{c i j}=\left(\sigma_{x}\right)_{i j}-\frac{\lambda}{4} \tilde{G}_{s}\left(\boldsymbol{k}, k_{\|}\right)_{i j}
$$

\footnotetext{
${ }^{9}$ Diagrams with tadpoles (one-point functions) are cancelled due to the equation of motion. Namely, in calculating the 1PI free energy, an appropriate source term is introduced depending on $M$ so that the equation of motion is always satisfied.
} 


$$
\begin{gathered}
\tilde{G}_{d}=1-\frac{\lambda}{4} \tilde{G}_{t}\left(\boldsymbol{k}, k_{\|}\right), \\
\tilde{G}_{d^{\prime}}=1-\frac{\lambda}{4} \tilde{G}_{u}\left(\boldsymbol{k}, k_{\|}\right),
\end{gathered}
$$

where $\sigma_{x}$ is an $x$-component of the Pauli matrix and

$$
\begin{aligned}
\tilde{G}_{s}\left(\boldsymbol{k}, k_{\|}\right)_{i j}= & \int d^{2} \boldsymbol{r} d^{d-1} r_{\|} e^{-i\left(\boldsymbol{k} \cdot \boldsymbol{r}+i k_{\|} \cdot r_{\|}\right)} \\
& \times\left\langle\left[\bar{\phi}_{j} \phi_{j}\right]\left(\boldsymbol{r} ; r_{\|}\right)\left[\bar{\phi}_{i} \phi_{i}\right](\mathbf{0} ; 0)\right\rangle, \\
\tilde{G}_{t}\left(\boldsymbol{k}, k_{\|}\right)= & \int d^{2} \boldsymbol{r} d^{d-1} r_{\|} e^{-i\left(\boldsymbol{k} \cdot \boldsymbol{r}+i k_{\|} \cdot r_{\|}\right)} \\
& \times\left\langle\left[\bar{\phi}_{1} \bar{\phi}_{2}\right]\left(\boldsymbol{r} ; r_{\|}\right)\left[\phi_{1} \phi_{2}\right](\mathbf{0} ; 0)\right\rangle, \\
\tilde{G}_{u}\left(\boldsymbol{k}, k_{\|}\right)= & \int d^{2} \boldsymbol{r} d^{d-1} r_{\|} e^{-i\left(\boldsymbol{k} \cdot \boldsymbol{r}+i k_{\|} \cdot r_{\|}\right)} \\
& \times\left\langle\left[\bar{\phi}_{2} \phi_{1}\right]\left(\boldsymbol{r} ; r_{\|}\right)\left[\bar{\phi}_{1} \phi_{2}\right](\mathbf{0} ; 0)\right\rangle .
\end{aligned}
$$

Thus the resulting contributions to EE, including both of those from the propagators and vertices, are given by

$$
\begin{aligned}
S_{\text {prop\&vert }}^{2 \text { PI }}= & -\frac{V_{d-1}}{6}\left(\sum_{i=1}^{2} \int^{1 / \epsilon} \frac{d^{d-1} k_{\|}}{(2 \pi)^{d-1}} \log \left[\tilde{G}_{\phi_{i}}^{-1}\left(\mathbf{0} ; k_{\|}\right) \epsilon^{2}\right]\right. \\
& -\frac{1}{2} \int^{1 / \epsilon} \frac{d^{d-1} k_{\|}}{(2 \pi)^{d-1}} \operatorname{tr} \log \left[\sigma_{x}-\frac{\lambda}{4} \tilde{G_{s}}\left(\mathbf{0} ; k_{\|}\right)\right] \\
& -\int^{1 / \epsilon} \frac{d^{d-1} k_{\|}}{(2 \pi)^{d-1}} \log \left[1-\frac{\lambda}{4} \tilde{G}_{t}\left(\mathbf{0} ; k_{\|}\right)\right] \\
& \left.-\int^{1 / \epsilon} \frac{d^{d-1} k_{\|}}{(2 \pi)^{d-1}} \log \left[1-\frac{\lambda}{4} \tilde{G}_{u}\left(\mathbf{0} ; k_{\|}\right)\right]\right)
\end{aligned}
$$

where the tr in the second line is a trace over the $2 \times 2$ matrix.

The above model is simple in the sense that the auxiliary field of each $s$, $t$, and $u$-channel is different and the correspondence between twisting a vertex and twisting propagator of each auxiliary field is clear. Let us then consider a less easy (though seemingly easier) case, namely the $\phi^{4}$-theory with a single real scalar. The action written with an auxiliary field $c$ takes the following form:

$$
I_{\text {stu }}=\int \frac{d^{d+1} x}{M}\left[\frac{1}{2} \phi\left(-\square+m_{0}^{2}\right) \phi+\frac{1}{2} c^{2}+i \sqrt{\frac{\lambda}{2}} c \phi^{2}\right] .
$$

In order to reproduce the vertex contributions to $\mathrm{EE}$ in the original $\lambda \phi^{4} / 4$ theory, we need to sum all the contributions from the three different channels for $c$. If we use the above action, the free energy in flat space can be reproduced, but not the free energy of the orbifold theory. Thus we cannot use the renormalized two-point function of $c$ via $\log G_{c}^{-1}$ to express the correct amount of vertex contributions to EE. $\mathrm{EE}$ in $\phi^{4}$ theory is neither expressed by a single auxiliary field $c$ nor by triple copies of it because the three channels coincide and get mixed among them.

In spite of this difficulty, we can still get a consistent description of vertex contributions, not through the auxiliary field method, but directly in terms of the composite operator. As the previous observation indicates, we will now focus on the following correlation function,

$$
G_{s t u}(x-y):=\left\langle: \phi^{2}:(x): \phi^{2}:(y)\right\rangle .
$$

The vertex contributions to EE in the $\phi^{4}$ theory is expected to be given by

$S_{\text {vert }}^{2 \mathrm{PI}}=\frac{V_{d-1}}{12} \int^{1 / \epsilon} \frac{d^{d-1} k_{\|}}{(2 \pi)^{d-1}} \log \left[1-\frac{3}{2} \lambda \tilde{G}_{\text {stu }}\left(\mathbf{0}, k_{\|}\right)\right]$.

Here, the coefficient $-3 \lambda / 2$ is understood as $-\lambda / 4 \times 6$ where $-\lambda / 4$ is the coefficient in front of the interaction vertex [the same coefficient as in Eq. (73)] and the coefficient 6 is the combinatorial factor for separating four $\phi(x)$ 's into a pair of two $\phi(y)$ 's. The unity in the logarithm in Eq. (76) means that the composite operator does not have any new degrees of freedom in the free field limit and does not contribute to EE. The overall factor is not $1 / 6$ but $1 / 12$ since the composite operator is real.

Since we cannot introduce the auxiliary field and use the conventional 2PI formalism, we do not yet know how to prove that the above expression of Eq. (76) gives the correct vertex contributions to EE. Instead, we will perturbatively check its correctness up to $\lambda^{2}$ in the following. The two-point function of the composite operator can be evaluated as

$$
G_{s t u}=2 A-6 \lambda A^{2}-12 \lambda B+O\left(\lambda^{2}\right),
$$

where

$A:=G_{0}(x-y)^{2}$,

$B:=\int d^{d+1} z G_{0}(x-y) G_{0}(x-z) G_{0}(z-y) G_{0}(0)$.

In Eq. (77), the product of operators represents a convolution; $X Y(x-y)=\int d^{d+1} z X(x-z) Y(z-y)$. By substituting Eq. (77) into Eq. (76), and using the identity

$$
\int \frac{d^{d-1} k_{\|}}{(2 \pi)^{d-1}} \tilde{f}\left(\mathbf{0}, k_{\|}\right)=\int d^{2} \boldsymbol{r} f(\boldsymbol{r}, 0),
$$

we can expand Eq. (76) up to $O\left(\lambda^{2}\right)$ as 


$$
\begin{aligned}
S_{\text {vert }}^{2 \text { PI }}= & \frac{V_{d-1}}{12} \int d^{2} \boldsymbol{r}\left[\log \left(1-\frac{3}{2} \lambda G_{\text {stu }}\right)\right](\boldsymbol{r}, 0) \\
= & \frac{V_{d-1}}{12} \int d^{2} \boldsymbol{r}\left[-\frac{3}{2} \lambda G_{s t u}-\frac{9}{8} \lambda^{2} G_{s t u}^{2}\right](\boldsymbol{r}, 0)+\mathcal{O}\left(\lambda^{3}\right) \\
= & \frac{V_{d-1}}{12} \int d^{2} \boldsymbol{r}\left[-3 \lambda A+18 \lambda^{2} B+\frac{9}{2} \lambda^{2} A^{2}\right](\boldsymbol{r}, 0)+\mathcal{O}\left(\lambda^{3}\right) \\
= & -\frac{V_{d-1}}{4} \lambda \int d^{2} \boldsymbol{r} G_{0}(\boldsymbol{r}, 0)^{2}+\frac{3 V_{d-1}}{2} \lambda^{2} \int d^{2} \boldsymbol{r} d^{2} \boldsymbol{s} d^{d-1} r_{\|} G_{0}(\boldsymbol{r}, 0) G_{0}\left(\boldsymbol{r}-\boldsymbol{s}, r_{\|}\right) G_{0}\left(\boldsymbol{s}, r_{\|}\right) G(\mathbf{0}, 0) \\
& +\frac{3 V_{d-1}}{8} \lambda^{2} \int d^{2} \boldsymbol{r} d^{2} \boldsymbol{s} d^{d-1} r_{\|} G_{0}\left(\boldsymbol{r}, r_{\|}\right)^{2} G_{0}\left(\boldsymbol{s}, r_{\|}\right)^{2}+\mathcal{O}\left(\lambda^{3}\right) .
\end{aligned}
$$

These three terms indeed coincide with Eqs. (54), (56), and (58), respectively.

The present result is surprising, or rather amusing since the non-Gaussian contributions to EE can be understood in terms of two-point functions of composite operators even when the auxiliary field can not be consistently introduced. As explained in Sec. IVA, a twisted propagator is pinned with loose ends reflecting quantum correlations between two spacial regions. From this observation, it is tempting to expect that EE can be interpreted as a sum of correlations of various composite operators, not restricted to those that appear at the classical action. Indeed, in the framework of the Wilsonian RG, the effective action (EA) changes as the energy scale is changed, and the EA contains infinitely many vertices. Thus EE will also follow the same RG flow. We want to come back to this important issue in the near future.

\section{GENERALIZATIONS TO THEORIES WITH SPINS}

All the above studies have been focused on scalar field theories. The analysis can be easily extended to a scalar theory with multiple flavors. Furthermore, we can straightforwardly extend it to general field theories with spins. As briefly explained at the end of Sec. II, we need an additional phase rotation corresponding to its spin. Besides a modification necessary for fermionic fields and subtlety for higher spin fields of $s \geq 3 / 2$, the orbifold method is applicable to them.

A twisted propagator with a spin- $s$ field $\varphi_{s}$ is accompanied with a rotation in the internal space:

$$
G_{\varphi_{s} 0}^{(M)}(x, y)=\sum_{m=0}^{M-1} e^{-2 i \theta_{m} \mathcal{M}_{1, d+1}^{(s)}} G_{\varphi_{s} 0}\left(\hat{g}^{m} x-y\right) .
$$

Here, $\mathcal{M}_{1, d+1}^{(s)}$ is one of the generators of $S O(d+1)$ in the spin- $s$ representation, which drives a rotation on a plane spanned by $x_{\perp}$ (1-direction) and $\tau((d+1)$-direction). For example, the propagator for a Dirac fermion is given by

$$
\begin{aligned}
S^{(M)}(x, y)= & \sum_{m=0}^{M-1} e^{\theta_{m} \gamma_{1} \gamma_{d+1}} \int \frac{d^{2} \boldsymbol{k}}{(2 \pi)^{2}} \frac{d^{d-1} k_{\|}}{(2 \pi)^{d-1}} \\
& \times \frac{i \boldsymbol{k} \cdot \boldsymbol{\gamma}+i k_{\|} \cdot \gamma_{\|}-m_{0}}{k^{2}+m_{0}^{2}} e^{i\left(\boldsymbol{k} \cdot \hat{g}^{m} \boldsymbol{x}-\boldsymbol{k} \cdot \boldsymbol{y}+k_{\|} \cdot\left(x_{\|}-y_{\|}\right)\right)}
\end{aligned}
$$

with $\gamma=\left(\gamma_{1}, \gamma_{d+1}\right)$ and $\gamma_{\|}=\left(\gamma_{2}, \ldots, \gamma_{d-2}\right)$.

In a bubble diagram, each propagator has such an additional rotational factor. However, since an interaction vertex is rotationally invariant, it is still invariant under $\mathbb{Z}_{M}$ rotation and consequently invariant under an overall twist of the adjacent propagators. ${ }^{10}$ Suppose that we have a multipoint vertex of fields with spins $s_{q}(q=1,2, \ldots)$ and the coefficient is given by $C_{i_{1} i_{2} \ldots}$. The $\mathbb{Z}_{M}$ invariance of the vertex is written as

$$
\begin{aligned}
& C_{i_{1} i_{2} \ldots} \delta^{2}\left(\boldsymbol{p}_{1}+\boldsymbol{p}_{2} \cdots\right)=\left(e^{2 i \theta_{m} \mathcal{M}_{1, d+1}^{\left(s_{1}\right)}}\right)_{i_{1}}{ }^{j_{1}}\left(e^{2 i \theta_{m} \mathcal{M}_{1, d+1}^{\left(s_{2}\right)}}\right)_{i_{2}}{ }^{j_{2}} \ldots \\
& \times C_{j_{1} j_{2} \ldots} \delta^{2}\left(\hat{g}^{m}\left(\boldsymbol{p}_{1}+\boldsymbol{p}_{2} \cdots\right)\right) \text {. }
\end{aligned}
$$

By decomposing each field into irreducible representations of $S O(2)$, this simply means that a sum of $S O(2)$ spins vanish at each vertex. Due to the invariance, the basic framework of $\mathbb{Z}_{M}$ gauge theory on Feynman diagrams is not changed. Namely, we can classify $\mathbb{Z}_{M}$ invariant configurations of twists in terms of fluxes in plaquettes as before. The additional phase associated with spins can be calculated by taking a special gauge of $\mathbb{Z}_{M}$ fluxes because of their gauge invariance.

Another point to notice is that, for fermions, we have to replace the twist operator $\hat{g}$ with $\hat{g}^{2}$ due to the antiperiodic boundary condition. In this case, $M$ should be considered as an odd integer.

\footnotetext{
${ }^{10} \mathrm{~A}$ simple example is a vertex in the $U(1)$ gauge theory, $\left(\gamma_{\mu}\right)_{\alpha \beta}$. It has one vector field and two spinor fields and is invariant under simultaneous rotations of the fields.
} 
In presence of higher spin fields, we can repeat the 2PI analysis. As far as the contributions from the propagators and vertices are concerned, it is sufficient to consider a twist of a particular propagator (or composite operator), and the additional phase can be easily obtained. For a general bosonic or fermionic field $\varphi_{s}$ with spin $s$, we can formally write down the free energy:

$$
\begin{aligned}
& \tilde{F}_{\varphi_{s}, \text { prop }}^{2 \text { PI }}=\frac{V_{d-1}}{2 M} \sum_{m=1}^{M-1} \frac{1}{4 \sin ^{2} \theta_{m}} \operatorname{tr}\left[e^{2 i \theta_{m} \mathcal{M}_{1, d+1}^{(s)}} \int \frac{d^{d-1} k_{\|}}{(2 \pi)^{d-1}} \log \tilde{G}_{\varphi_{s}}\left(\mathbf{0}, k_{\|}\right)\right] \quad \text { (for bosons), } \\
& \tilde{F}_{\varphi_{s}, \text { prop }}^{2 \text { PI }}=-\frac{V_{d-1}}{2 M} \sum_{m=1}^{M-1} \frac{1}{4 \sin ^{2} 2 \theta_{m}} \operatorname{tr}\left[e^{4 i \theta_{m} \mathcal{M}_{1, d+1}^{(s)}} \int \frac{d^{d-1} k_{\|}}{(2 \pi)^{d-1}} \log \tilde{G}_{\varphi_{s}}\left(\mathbf{0}, k_{\|}\right)\right] \text {(for fermions). }
\end{aligned}
$$

"tr" here represents the trace over the internal space. Moreover, the vertex contributions are written in terms of the renormalized propagators of composite operators as well as the scalar field case. When one considers a general composite operator such as $: \varphi_{s} \varphi_{s^{\prime}}^{\prime}:$, it is generically in a reducible representation of $S O(d+1)$. We first decompose it into irreducible components, each of which corresponds to a different composite operator.

If we reduce Eqs. (84), (85) to the free field cases, we can easily evaluate the trace because both the rotational factor and $\tilde{G}_{\varphi 0}\left(\mathbf{0} ; k_{\|}\right)$are diagonalized in the basis of the eigenstates for $S O(2)$. The resulting EEs coincide with those in [62]. On the other hand, for interacting cases, $\tilde{G}_{\varphi}\left(\mathbf{0} ; k_{\|}\right)$has off-diagonal components and we need to take a trace of the product of the rotational factor and the matrixvalued logarithmic terms in a nontrivial way. It is technically difficult to proceed to further computations and we leave it for future investigations. Meanwhile, we can conclude that the non-Gaussian part in $\mathrm{EE}$ is understood as contributions from renormalized two-point functions of composite operators while the Gaussian part is a contribution from the fundamental fields.

\section{CONCLUSIONS AND DISCUSSIONS}

In the present paper, we have studied EE in general interacting QFTs from the field theoretical perspective proposed in our previous work [58]. The approach is based on the orbifold method to calculate EE of half space and the consequent idea of $\mathbb{Z}_{M}$ gauge theory on Feynman diagrams. In this method, EE is given by a sum of various configurations of $\mathbb{Z}_{M}$ fluxes on each of the plaquettes in Feynman diagrams. Among infinitely many configurations of fluxes, we have extracted two dominant contributions to $\mathrm{EE}$, that correspond to twisting propagators and vertices. An essential development in the present paper from our previous work [58] is a new interpretation of the vertex contributions in terms of correlation functions of composite operators. We have also shown that the propagator contributions to EE are exactly given by the full renormalized propagators in the 2PI formalism where two-point functions are treated nonperturbatively, and as a consequence, we have succeeded to fully extract the Gaussian contributions to EE, Thus the vertex contributions that are interpreted as correlations of composite operators purely represent the non-Gaussianity of the vacuum.

Then, one of the most crucial questions left unanswered is how we can understand or evaluate configurations of twists other than those corresponding to a single twisted propagator or vertex. We might be able to address this question by developing an efficient computational method in the $\mathbb{Z}_{M}$ gauge theory on Feynman diagrams. On the other hand, we may be able to extract further contributions by extending our finding that some of the non-Gaussian contributions are interpreted as correlations of composite operators. Suppose that a bubble diagram is separated into two pieces connected by a fat propagator. Then, twisting the fat propagator might give a contribution to EE associated with the correlation of the macroscopic composite operators. It is tempting to expect that a general configuration of twists would be understood as a correlation of various composite operators between two spatial regions. If this expectation is true, then what quantity of composite operators will give the magnitude of its contributions to EE? A naive guess is its mass dimension or the correlation length. In our studies, we have extracted contributions of the propagator and the vertices. The propagator contribution to $\mathrm{EE}$ is given by scalar fields with the mass dimension 1 , while the vertex contributions are given by composite operators with the mass dimension 2. It is the reason why we think that they give dominant contributions to $\mathrm{EE}$ compared to others.

We may also apply the method of Wilsonian RG to extract further contributions to EE. In the present paper, we have considered such vertex contributions that the vertices are already present in the classical action. In the Wilsonian RG picture, the effective action is scale-dependent and contains many other vertices besides those present in the classical action. Then we may introduce further auxiliary fields corresponding to various composite operators whose mass dimensions are higher than 2. Since these composite operators are expected to decay faster than those studied in 
the present paper, their contributions will be less dominant but we may be able to extract contributions to $\mathrm{EE}$ systematically by using the Wilsonian effective action.

Along the Wilsonian RG flow, quantum vertices appear in addition to classical vertices while the dynamical degrees of freedom to be integrated decrease. From this perspective, we can partly answer the unsolved questions: Do the other configurations of twists not discussed in Sec. IV and V contribute? For instance, a general flux configuration of the figure-eight 2-loop diagram is characterized by two $\mathbb{Z}_{M}$ integers while only $(m, 0),(0, m)$, and $(m,-m)$ can be identified as twists of propagators or vertices (Fig. 10). The fluxes other than these special sets should be regarded as twisting more than one propagator or vertex and cannot be attributed to a single propagator or vertex. Thus we could not evaluate such contributions. However, this problem at the original UV theory is no more a problem in the IR effective theory. After renormalization, such configurations of fluxes in the IR limit are either interpreted as twisting general vertices, which implies twisting more general composite operators, or abandoned as the UV part of contributions and absent in the IR universal part. They should be treated in the Wilsonian effective field theory and now under investigation [71].

Furthermore, the Wilsonian RG approach will tell us how we should take the renormalization scale in the calculation of $\mathrm{EE}$ and also how $\mathrm{EE}$ varies along the $\mathrm{RG}$ flow. In relation to this, it is worthwhile to investigate how our results are connected to another method using the continuous multiscale entanglement renormalization ansatz $[42,43,72]$.

It is also interesting to generalize our results to other choices of spatial subregions. Our investigation depends heavily on the orbifold method, which is not applicable for regions other than the half space of flat space. However, the results and observations have general implications based on two-point functions pinned at the boundary. As we have shown, twisted propagators are pinned at the boundary, which can be interpreted as reducing the degrees of freedom in the normal direction to the boundary. If the boundary has a more nontrivial shape, we need a clever way to foliate the space to specify correlations between such two spacial regions. It is tempting to relate it to the holographic view of EE [10-12], or a geometric perturbation [21].

Other generalizations include EE in nonrelativistic QFTs or EE of excited states. Since our approach uses a standard QFT technique of Feynman diagrams, it should be relatively easy to study EE of excited states. For example, EE of an excited state by operators $O$ must be obtained by replacing the free energy with the corresponding correlation functions $\left\langle O_{1}^{\dagger} O_{1} \cdots O_{M}^{\dagger} O_{M}\right\rangle$, where $O_{i}$ is given by $O$ restricted within the half of the $i$ th piece of the orbifolded space. In CFTs, some concrete calculations based on this approach are performed and applied to the orbifold method [73] as well as the standard replica trick [74-82]. As for a practical application, we could explicitly investigate the entropic c-theorem [83] along the RG flow based on the direct computation of $\mathrm{EE}$ in the presence of interactions.

\section{ACKNOWLEDGMENTS}

We are supported in part by JSPS Grant-in-Aid for Scientific research, No. 18H03708 (S. I.), No. 16H06490 (S. I.), No. 20J00079 (K. S.).
[1] T. J. Osborne and M. A. Nielsen, Phys. Rev. A 66, 032110 (2002).

[2] A. Osterloh, L. Amico, G. Falci, and R. Fazio, Nature (London) 416, 608 (2002).

[3] G. Vidal, J. I. Latorre, E. Rico, and A. Kitaev, Phys. Rev. Lett. 90, 227902 (2003).

[4] B.-Q. Jin and V. E. Korepin, J. Stat. Phys. 116, 79 (2004).

[5] C. Holzhey, F. Larsen, and F. Wilczek, Nucl. Phys. B424, 443 (1994).

[6] P. Calabrese and J. Cardy, J. Stat. Mech. (2004) P06002.

[7] A. Kitaev and J. Preskill, Phys. Rev. Lett. 96, 110404 (2006).

[8] M. Levin and X.-G. Wen, Phys. Rev. Lett. 96, 110405 (2006).

[9] J. P. Ibieta-Jimenez, M. Petrucci, L. N. Queiroz Xavier, and P. Teotonio-Sobrinho, J. High Energy Phys. 03 (2020) 167.

[10] S. Ryu and T. Takayanagi, Phys. Rev. Lett. 96, 181602 (2006).
[11] S. Ryu and T. Takayanagi, J. High Energy Phys. 08 (2006) 045.

[12] V. E. Hubeny, M. Rangamani, and T. Takayanagi, J. High Energy Phys. 07 (2007) 062.

[13] S. N. Solodukhin, Living Rev. Relativity 14, 8 (2011).

[14] A. Almheiri, T. Hartman, J. Maldacena, E. Shaghoulian, and A. Tajdini, arXiv:2006.06872.

[15] A. Almheiri, R. Mahajan, J. Maldacena, and Y. Zhao, J. High Energy Phys. 03 (2020) 149.

[16] G. Penington, S. H. Shenker, D. Stanford, and Z. Yang, arXiv:1911.11977.

[17] Y. Nambu, Phys. Rev. D 78, 044023 (2008).

[18] P. Ruggiero, E. Tonni, and P. Calabrese, J. Stat. Mech. (2018) 113101.

[19] L.-Y. Hung, R. C. Myers, and M. Smolkin, J. High Energy Phys. 10 (2014) 178.

[20] H. Casini and M. Huerta, Phys. Lett. B 694, 167 (2010). 
[21] V. Rosenhaus and M. Smolkin, J. High Energy Phys. 02 (2015) 015.

[22] V. Rosenhaus and M. Smolkin, J. High Energy Phys. 12 (2014) 179.

[23] V. Rosenhaus and M. Smolkin, J. High Energy Phys. 09 (2014) 119.

[24] T. Nishioka, S. Ryu, and T. Takayanagi, J. Phys. A 42, 504008 (2009).

[25] T. Nishioka, Rev. Mod. Phys. 90, 035007 (2018).

[26] A. Botero and B. Reznik, Phys. Rev. A 70, 052329 (2004).

[27] D. Katsinis and G. Pastras, Eur. Phys. J. C 78, 282 (2018).

[28] E. Bianchi and A. Satz, Phys. Rev. D 99, 085001 (2019).

[29] A. Lewkowycz, R. C. Myers, and M. Smolkin, J. High Energy Phys. 04 (2013) 017.

[30] H. Casini and M. Huerta, J. Phys. A 42, 504007 (2009).

[31] M. P. Hertzberg and F. Wilczek, Phys. Rev. Lett. 106, 050404 (2011).

[32] C. P. Herzog and T. Nishioka, J. High Energy Phys. 03 (2013) 077.

[33] W. Donnelly, S. Timmerman, and N. Valdés-Meller, J. High Energy Phys. 04 (2020) 182.

[34] D. L. Jafferis, I. R. Klebanov, S. S. Pufu, and B. R. Safdi, J. High Energy Phys. 06 (2011) 102.

[35] S. S. Pufu, J. Phys. A 50, 443008 (2017).

[36] T. Nishioka and I. Yaakov, J. High Energy Phys. 10 (2013) 155.

[37] M. P. Hertzberg, J. Phys. A 46, 015402 (2013).

[38] Y. Chen, L. Hackl, R. Kunjwal, H. Moradi, Y. K. Yazdi, and M. Zilhão, J. High Energy Phys. 11 (2020) 114.

[39] M. A. Metlitski, C. A. Fuertes, and S. Sachdev, Phys. Rev. B 80, 115122 (2009).

[40] C. Akers, O. Ben-Ami, V. Rosenhaus, M. Smolkin, and S. Yankielowicz, J. High Energy Phys. 03 (2016) 002.

[41] J. Cotler and M. T. Mueller, Ann. Phys. (Amsterdam) 365, 91 (2016).

[42] J. J. Fernandez-Melgarejo and J. Molina-Vilaplana, J. High Energy Phys. 02 (2021) 106.

[43] J. J. Fernández-Melgarejo and J. Molina-Vilaplana, arXiv: 2104.01551.

[44] A. Bhattacharyya, L.-Y. Hung, and C. M. Melby-Thompson, J. High Energy Phys. 10 (2017) 081.

[45] L. Wang and M. Troyer, Phys. Rev. Lett. 113, 110401 (2014).

[46] P. V. Buividovich and M. I. Polikarpov, Nucl. Phys. B802, 458 (2008).

[47] P. V. Buividovich and M. I. Polikarpov, Phys. Lett. B 670, 141 (2008).

[48] E. Itou, K. Nagata, Y. Nakagawa, A. Nakamura, and V. I. Zakharov, Prog. Theor. Exp. Phys. (2016), 061B01.

[49] A. Rabenstein, N. Bodendorfer, A. Schäfer, and P. Buividovich, Phys. Rev. D 100, 034504 (2019).

[50] S. Whitsitt, W. Witczak-Krempa, and S. Sachdev, Phys. Rev. B 95, 045148 (2017).

[51] H. R. Hampapura, A. Lawrence, and S. Stanojevic, Phys. Rev. B 100, 134412 (2019).
[52] J. H. Cooperman and M. A. Luty, J. High Energy Phys. 12 (2014) 045.

[53] T. Barrella, X. Dong, S. A. Hartnoll, and V. L. Martin, J. High Energy Phys. 09 (2013) 109.

[54] M. Taylor and W. Woodhead, J. High Energy Phys. 08 (2016) 165.

[55] M. Taylor and L. Too, J. High Energy Phys. 12 (2020) 050.

[56] H. Liu and M. Mezei, J. High Energy Phys. 04 (2013) 162.

[57] H. Liu and M. Mezei, J. High Energy Phys. 01 (2014) 098.

[58] S. Iso, T. Mori, and K. Sakai, Phys. Rev. D 103, 105010 (2021).

[59] J. M. Cornwall, R. Jackiw, and E. Tomboulis, Phys. Rev. D 10, 2428 (1974).

[60] J. Berges, AIP Conf. Proc. 739, 3 (2004).

[61] T. Nishioka and T. Takayanagi, J. High Energy Phys. 01 (2007) 090.

[62] S. He, T. Numasawa, T. Takayanagi, and K. Watanabe, J. High Energy Phys. 05 (2015) 106.

[63] G. von Gersdorff, J. High Energy Phys. 08 (2008) 097.

[64] A. Gervois and M. L. Mehta, J. Math. Phys. (N.Y.) 36, 5098 (1995).

[65] J. Eisert, M. Cramer, and M. B. Plenio, Rev. Mod. Phys. 82, 277 (2010).

[66] N. Shiba and T. Takayanagi, J. High Energy Phys. 02 (2014) 033.

[67] T. He, J. M. Magan, and S. Vandoren, SciPost Phys. 3, 034 (2017).

[68] M. R. Mohammadi Mozaffar and A. Mollabashi, J. High Energy Phys. 07 (2017) 120.

[69] S. A. Gentle and S. Vandoren, J. High Energy Phys. 07 (2018) 013.

[70] N. Ogawa, T. Takayanagi, and T. Ugajin, J. High Energy Phys. 01 (2012) 125.

[71] S. Iso, T. Mori, and K. Sakai, arXiv:2105.14834.

[72] J. J. Fernandez-Melgarejo and J. Molina-Vilaplana, J. High Energy Phys. 07 (2020) 149.

[73] P. Caputa, Y. Kusuki, T. Takayanagi, and K. Watanabe, J. Phys. A 50, 244001 (2017).

[74] M. Nozaki, T. Numasawa, and T. Takayanagi, Phys. Rev. Lett. 112, 111602 (2014).

[75] M. Nozaki, J. High Energy Phys. 10 (2014) 147.

[76] P. Caputa, M. Nozaki, and T. Takayanagi, Prog. Theor. Exp. Phys. (2014), 093B06.

[77] M. Nozaki, T. Numasawa, and S. Matsuura, J. High Energy Phys. 02 (2016) 150.

[78] B. Chen, W.-Z. Guo, S. He, and J.-q. Wu, J. High Energy Phys. 10 (2015) 173.

[79] S. He, T. Numasawa, T. Takayanagi, and K. Watanabe, Phys. Rev. D 90, 041701(R) (2014).

[80] P. Caputa and A. Veliz-Osorio, Phys. Rev. D 92, 065010 (2015).

[81] T. Numasawa, J. High Energy Phys. 12 (2016) 061.

[82] M. Nozaki and N. Watamura, J. High Energy Phys. 12 (2016) 069.

[83] H. Casini and M. Huerta, Phys. Lett. B 600, 142 (2004). 